\title{
43. GEOCHEMICAL STUDIES ON SELECTED SEDIMENT SAMPLES FROM THE LAU BACKARC BASIN: EVIDENCE FOR HYDROTHERMAL PONDED SEDIMENTS ${ }^{1}$
}

\author{
Gérard Blanc ${ }^{2}$
}

\begin{abstract}
Major and minor ( $\mathrm{Mn}, \mathrm{Sr}, \mathrm{Ba}, \mathrm{V}, \mathrm{Cr}, \mathrm{Ni}, \mathrm{Co}, \mathrm{Zn}, \mathrm{Cu}, \mathrm{Zr}, \mathrm{Y}, \mathrm{Sc})$ elements and mineralogic compositions were determined on bulk sediments collected during Ocean Drilling Program Leg 135. Three classes of sediment samples from holes drilled in the Lau Basin are discriminated by mineralogy and major element data. Samples labeled Class 1 are significantly enriched in biogenic calcite and occur predominantly in the northern part of the basin (Sites 834-835), whereas those of Class 3 are mostly enriched in volcanogenic material and are predominant in the central part of the basin (Sites 836-839).

The minor element composition records the effects of the hydrothermal activity on the sediments. In the northern area of the basin (Sites 834-835), sedimentation is characterized by higher accumulation rates of the carbonate and hydrothermal fractions. These sediments are probably reworked predominantly, transported in the water column, and then settled locally. Thus, ponded sediments are probably responsible to this high accumulation rates. Diagenetic processes altered the volcanic material to a grade corresponding to the stability of phillipsite. In the central area of the basin (Sites 836-839), sedimentation is characterized by the action of bottom currents preferentially reworking the carbonate and hydrothermal fractions. Volcanogenic accumulation rates are greater at these sites than in the northern Lau Basin. Alteration of volcanic material is more important deeper in the holes and records authigenesis of clay rich in $\mathrm{Fe}-\mathrm{Mg}$, most likely smectite. Locally, clay minerals have apparently incorporated $\mathrm{Cr}$ and other ore-forming elements.
\end{abstract}

\section{INTRODUCTION}

During Leg 135, the JOIDES Resolution recovered sediments from two areas (northern and central) located west of the Central Lau (CLSC) and Eastern Lau (ELSC) spreading centers. The western area of the Lau Basin is characterized by a number of small, elongate, north-trending sedimentary basins and is bounded on the west by the islands and atoll reefs of the Lau Ridge. Sites 834 and 835 were drilled in two northern separate basins between 100 and $200 \mathrm{~km}$ west of CLSC, whereas Sites 836-839 lie in small central basins closer (from 50 to $80 \mathrm{~km}$ ) to the ELSC (Fig. 1). These backarc sites can be divided, therefore, into those distal to the CLSC (Sites 834-835) and proximal to the ELSC (Sites 836-839). Previous geochemical data for sediments from the Lau Basin are limited to material younger than 700 k.y. (Riech, 1990). Leg 135 data indicate that sedimentation in the Lau Basin began after formation of backarc basin crust at each site. The basement ages range from approximately $4 \mathrm{Ma}$ (Site 834 ) to 3 $\mathrm{Ma}$ (Site 835) for the northern sites (834-835), whereas those of the basement from southern sites (836-839) range between $0.6 \mathrm{Ma}$ (Site 836 ) and $2.1 \mathrm{Ma}$ (Site 837 ). The sedimentary sequences recovered in the Lau Basin consist predominantly of clay and pelagic nannofossil ooze interbedded with volcanic material and overlying redeposited volcaniclastics. The volcaniclastic units at Sites 836-839 are much thicker and coarser grained than the sediments recovered at Sites 834 835 . The sedimentation rates range from $4 \mathrm{~mm} / \mathrm{ka}$ to $88 \mathrm{~mm} / \mathrm{ka}$. The average content of organic carbon in the sediments is $0.14 \%$. This carbon is mostly inertinite with no metabolic value for bacteria. (Parson, Hawkins, Allan, et al., 1992).

The above presented sites provide an ideal location to study the mineralogy and the chemistry of bulk sediments adjacent to an active spreading center. On the basis of the rather constant composition of the hydrothermal precipitates that diagenetic reprecipitation and formation of layers rich in Mn oxides have been shown to be of minor importance

\footnotetext{
'Hawkins, J., Parson, L., Allan, J., et al., 1994. Proc. ODP. Sci. Resuits, 135: College Station, TX (Ocean Drilling Program).

${ }^{2}$ Centre de Sédimentologie et de Géochemie de la Surface, CNRS UPR no. 6251, Institut de Géologie Strasbourg (IGS), 1 rue Blessig, 67084 Strasbourg Cedex, France.
}

in the Lau Basin during the last 700 k.y. (Riech, 1990). All clay and pelagic nannofossil oozes, which were recovered at the backarc sites, are stained by a distinctive reddish brown color because of the oxyhydroxides, which are probably hydrothermally derived (Parson, Hawkins, Allan, et al., 1992). The aim of the present paper is to characterize mineralogically and chemically the sediment of the Leg 135 Lau Basin, to determine if similarities exist between the chemistry of Lau Basin sediments and that of sediments from the flanks of other Pacific Ocean spreading ridges, and to characterize the influence of hydrothermal activity on the sediment composition as a function of parameters such as time and distance from the spreading centers of the Lau Basin.

\section{METHODS}

All bulk sediment samples were powdered in a carbon steel mill, dried at $110^{\circ}(\approx 12 \mathrm{hr})$, and then ashed at $1000^{\circ} \mathrm{C}$ for $3 \mathrm{hr}$. Loss-onignition measurements resulted from the weight difference between dried and ashed samples. Sample splits of $100 \mathrm{mg}$ were fused for 20 minutes with $750 \mathrm{mg}$ of pure lithium tetraborate under inert Ar atmosphere in a crucible (Carbone Lorraine V25), and were dissolved as molten fusion product in a glycerin-hydrochloric acid solvent. Addition of glycerin increases the solubility of borate acid and stabilizes the solution viscosity, consequently increasing the reproducibilities of the flux injection and of the intensity of the spectrum lines (Samuel et al., 1985). The 1:250 final dilution is introduced into the spectrometers. Concentrations of $\mathrm{Na}$ and $\mathrm{K}$ were determined on a Corning Flam emission spectrometer, the other major elements were determined on a ARL 14000 arc spectrometer (Besnus and Rouault, 1973). Mn, Sr, $\mathrm{Ba}, \mathrm{V}, \mathrm{Cr}, \mathrm{Ni}, \mathrm{Co}, \mathrm{Zn}, \mathrm{Cu}, \mathrm{Zr}$, and $\mathrm{Y}$ were analyzed using a ARL 35000 ICP-AES (inductively coupled plasma-atomic emission spectrometer). Accuracy of the analyses was determined by comparisons to marine sediment international standards previously analyzed at the CRPG, the ANRT, and the USGS: SD0I (East Pacific Rise Sediment), SD02 (Central Pacific Sediment), and MAG1 (Marine Mud). It is within 5\% for all determined elements. Precision of the analyses, as determined from duplication of the sample preparation, is between $2 \%$ and $5 \%$ for the major elements, and $10 \%$ for $\mathrm{P}_{2} \mathrm{O}_{5}$ in samples having concentrations lower than $0.20 \mathrm{wt} \%$. The precision is generally $5 \%$ for the minor elements at concentrations 10 times the detection limit and 


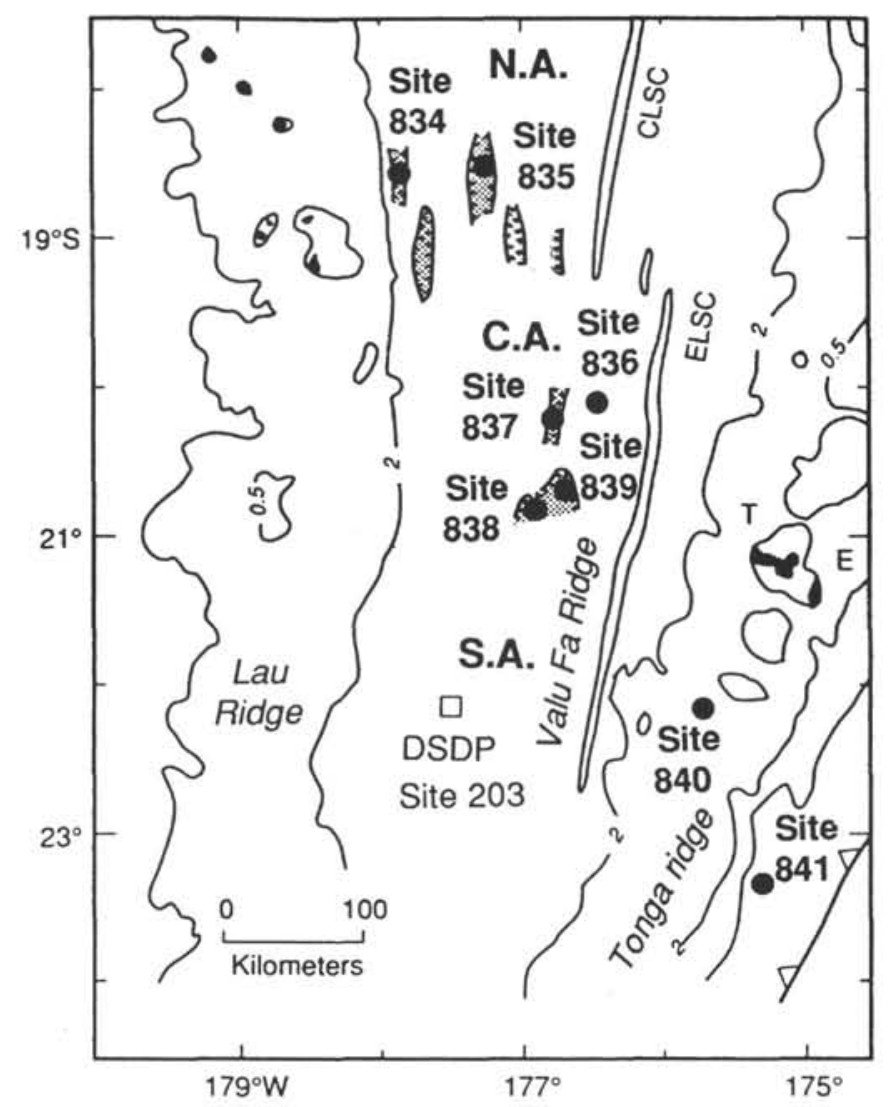

Figure 1. Map of backarc sites drilled in the Lau Basin during Leg 135 with outline bathymetry ( 500 and $2000 \mathrm{~m}$ isobaths). Contour interval in kilometers. Known sub-basins are stippled. ELSC $=$ East Lau Spreading Center and CLSC $=$ Central Lau Spreading Center. $\mathrm{T}=$ Tongatapu and $\mathrm{E}=$ 'Eua (from Parson, Hawkins, Allan, et al., 1992). N.A. = northern area, C.A. = central area, and S.A. $=$ southern area.

$20 \%$ for those at 5 times the detection limit, which gives a general precision of $5 \%$ for $\mathrm{Mn}, \mathrm{Sr}, \mathrm{Ba}, \mathrm{V}, \mathrm{Cr}, \mathrm{Zn}, \mathrm{Cu}, \mathrm{Sc}, \mathrm{Y}, \mathrm{Zr}$, and $20 \%$ for $\mathrm{Ni}$ and $\mathrm{Co}$. Analyses were made on 38 squeezed cake samples and on 22 selected samples that did not include exceptional features. The contents of the major elements are expressed in oxide weight per 100 $\mathrm{g}$ dried sample (wt\%; Table 1) and the contents of the minor elements are given in milligrams per kilogram (Table 2).

The mineralogical compositions of the major mineral phases were determined on dry powdered sediments by X-ray diffraction (XRD) techniques using a Phillips PW 1710. The samples were run between $3^{\circ}$ and $65^{\circ} 2 \theta$ at $40 \mathrm{kV} / 20 \mathrm{~mA}$, using $\mathrm{Cu} \mathrm{K} \alpha$ radiation, a Ni filter, and a scan speed of $1 \% \mathrm{~min}$. Mineral percentages were estimated from the peak intensity using mass absorption coefficients and chemical data (Hooton and Giorgetta, 1977).

\section{RESULTS AND DISCUSSION}

\section{Characterization of the Sediment Samples}

Comparison between major solid phases and major element chemistry allows characterization of the studied sediment. In Figures 2-6, the cumulative contents (in percent) of the major minerals and the amorphous silica, at each site, are compared with cumulative amounts (in oxide weight percent) of the major chemical elements and amount of loss-on-ignition $\left(1000^{\circ} \mathrm{C}\right)$. Calcite, clay minerals, and amorphous silica are the three dominant solid phases. For all samples, except for the aragonite-bearing sample $(135-834 \mathrm{~A}-1 \mathrm{H}-4,140-150 \mathrm{~cm})$ and for the analcime-bearing sample $(135-834 \mathrm{~A}-16 \mathrm{X}-1,140-150 \mathrm{~cm})$, the average value of calcite, clay minerals, and amorphous silica cumulative percent equal $94.6 \pm 3.4 \%$. The amount of calcite fits well with the $\mathrm{CaO}$ content $(r=0.991)$ and that of amorphous silica + clay with that of $\mathrm{SiO}_{2}(r=0.932)$ (Fig. 7). The proportion of $\mathrm{CaCO}_{3}$ calculated from total $\mathrm{Ca}$ content is comparable to the volumetric carbonate determinations obtained by XRD, and thus the errors resulting from the $\mathrm{Ca}$ content of any silicate present are less than the analytical uncertainty $(<5 \%)$. A contribution of noncarbonate $\mathrm{CaO}$ of $1.4 \mathrm{wt} \%$ can be estimated from the correlation line (i.e., calcite $=1.80 \mathrm{CaO}-$ $2.48)$. The presence of aragonite $(\approx 55 \%)$ in addition to calcite $(\approx 27 \%$ ) explains the high content of $\mathrm{CaO}(\approx 47.8 \mathrm{wt} \%)$ in Sample 135-834A$1 \mathrm{H}-4,140-150 \mathrm{~cm}$, whereas the occurrence of analcime $(\approx 25 \%)$, amphibole $(\approx 5 \%)$, and feldspar $(\approx 15 \%)$, in addition to clay minerals $(\approx 45 \%)$, explains the high content of $\mathrm{SiO}_{2}(\approx 59 \mathrm{wt} \%)$ in Sample $135-834 \mathrm{~A}-16 \mathrm{X}-1,140-150 \mathrm{~cm}$. For all other samples, comparisons between the solid phase content and the chemical composition allow differentiation of the following three classes of samples in the holes studied (Fig. 8):

1. Samples of Class 1: sediments rich in biogenic calcite;

2. Samples of Class 2: sediments rich in biogenic calcite and volcanogenic components;

3. Samples of Class 3: sediments rich in volcanogenic components.

These three classes of sediments are not independent chemical entities (Table 3 and Fig. 8), as the material of Class 2 appears to be intermediate between the two other classes, which approximate chemical end-members. Sample 135-835A-8H-4, 140-150 cm, seems to correspond to the calcite end-member of Class $1(\mathrm{CaO}=42.7 \mathrm{wt} \%$, $\mathrm{SiO}_{2}=7.6 \mathrm{wt} \%$ ) and Sample $135-837 \mathrm{~A}-9 \mathrm{H}-5,78-80 \mathrm{~cm}$, seems to correspond to the volcanogenic end-member of Class $3(\mathrm{CaO}=4.0$ $\mathrm{wt} \%, \mathrm{SiO}_{2}=63.6 \mathrm{wt} \%$ ). Class 1 samples occur predominantly in the northern drilling sites (834-835), whereas Class 3 samples are found predominantly in the southern drilling sites (838-839). Because $\mathrm{Ca}$ concentration is directly related to the total calcite content, by studying the relationships among $\mathrm{Ca}, \mathrm{Mg}$, and $\mathrm{Sr}, \mathrm{Mn}$ can then be used to obtain information about the distribution of the three former elements. In a $\mathrm{Mg}$ vs. Ca diagram (Fig. 9), samples of Class 3 can be clearly identified from the other classes. For samples from Classes 1 and 2, Mg correlates negatively with $\mathrm{Ca}(\mathrm{Mg}=-0.039 \mathrm{Ca}+1.84, r=0.729)$, whereas $\mathrm{Mg}$ correlates positively with $\mathrm{Ca}$ for the samples of Class $3(\mathrm{Mg}=0.42 \mathrm{Ca}$ $-0.44, r=0.854$ ). The $\mathrm{Mg} / \mathrm{Ca}$ ratio is $0.032 \pm 0.009$ for Class $1,0.071$ \pm 0.02 for Class 2 , and $0.28 \pm 0.09$ for Class 3 . The sample with the largest enrichment in $\mathrm{Ca}$ and calcite $(135-835 \mathrm{~A}-8 \mathrm{H}-4,140-150 \mathrm{~cm})$ has a $\mathrm{Mg} / \mathrm{Ca}$ ratio of 0.018 ; thus, the $\mathrm{Mg}$ enrichment of the samples of Classes 1 and 2 could be attributed to the $\mathrm{Mg}$ contents in the clay minerals. For Class 3, samples showing the lowest $\mathrm{Mg}$ and Ca contents are the most enriched in amorphous silica (hence, in volcanic glass), because of the minimal presence of the siliceous organisms. This suggests that the clay minerals are the major $\mathrm{Mg}$ sink and that calcite has a low amount of $\mathrm{Mg}$. For samples from Classes 1 and 2, Al correlates positively with $\mathrm{Si}(\mathrm{Al}=0.27 \mathrm{Si}+0.43, r=0.980)$ (Fig. 10). This simultaneous increase in $\mathrm{Al}$ and $\mathrm{Si}$ can be connected to the increase of volcanic glass, clays, feldspar, quartz, and zeolites in these samples. $\mathrm{Al}$ correlates negatively with $\mathrm{Si}$ for samples from Class $3(\mathrm{Al}=-0.31$ $\mathrm{Si}+15.5, r=0.770$ ). This could be explained by the higher content of the volcaniclastic material in these samples. Samples from deeper parts of Hole 839A (135-839A-18X-CC, 21-31 cm, -20X-CC, 1-3 cm, and $-21 \mathrm{X}-\mathrm{CC}, 12-14 \mathrm{~cm}$ ) are not considered in the correlation line because they are the most enriched in $\mathrm{Al}$ with respect to $\mathrm{Si}$, and also the most enriched in $\mathrm{Mg}$ relative to $\mathrm{Ca}$ (Table 1 and Fig. 9), which suggests that $\mathrm{Mg}$-rich clays occur in these samples.

$\mathrm{Sr}$ correlates positively with $\mathrm{Ca}$ (Fig. 11), and calcite is the major Ca sink. Hence, the greater volume of volcanic and/or hydrothermal silicates and oxides may dilute the normal calcite-bearing sediment, and samples from of Class 3 are easily identified in a Sr vs. Ca diagram because they have lower $\mathrm{Sr}$ and $\mathrm{Ca}$ concentrations (Fig. 11). Sample 
Table 1. Major element composition of bulk sediments, Holes 834A-839A.

\begin{tabular}{|c|c|c|c|c|c|c|c|c|c|c|c|c|}
\hline $\begin{array}{c}\text { Core, Section } \\
\text { Interval }(\mathrm{cm}) \\
\end{array}$ & $\begin{array}{l}\text { Depth } \\
\text { (mbsi) }\end{array}$ & $\begin{array}{c}\mathrm{SiO} 2 \\
\left(\mathrm{~W}_{\mathrm{t}} \%\right) \\
\end{array}$ & $\begin{array}{l}\mathrm{Al} 2 \mathrm{O} 3 \\
(\mathrm{~W} t \%)\end{array}$ & $\begin{array}{l}\mathrm{MgO} \\
(\mathrm{W}+\%)\end{array}$ & $\begin{array}{c}\mathrm{CaO} \\
(\mathrm{Wt} \%)\end{array}$ & $\begin{array}{l}\mathrm{Fe} 2 \mathrm{O} 3 \\
(\mathrm{Wt} \%)\end{array}$ & $\begin{array}{c}\mathrm{TiO} 2 \\
(\mathrm{Wt} \%)\end{array}$ & $\begin{array}{l}\text { P2O5 } \\
\left(W_{t} \%\right)\end{array}$ & $\begin{array}{l}\mathrm{Na} 2 \mathrm{O} \\
(\mathrm{Wt} \%) \\
\end{array}$ & $\begin{array}{l}\mathrm{K} 2 \mathrm{O} \\
(\mathrm{W} t \%) \\
\end{array}$ & $\begin{array}{r}\text { weight lost } \\
\text { at } 1000^{\circ} \mathrm{C}\end{array}$ & Total \\
\hline $135-834 \mathrm{~A}-1 \mathrm{H}-4,140-150$ & 5.9 & 4.3 & 1.0 & 1.89 & 47.8 & 1.2 & 0.06 & 0.17 & 0.10 & 0.05 & 42.26 & 99.46 \\
\hline $135-834 \mathrm{~A}-2 \mathrm{H}-4,140-150$ & 13.5 & 13.6 & 3.7 & 1.11 & 38.4 & 4.8 & 0.19 & 0.31 & 0.06 & 0.05 & 34.95 & 98.97 \\
\hline $135-834 \mathrm{~A}-3 \mathrm{H}-4,140-150$ & 23.0 & 16.3 & 3.9 & 1.06 & 37.4 & 3.4 & 0.21 & 0.27 & 0.07 & 0.05 & 34.98 & 99.21 \\
\hline $135-834 \mathrm{~A}-4 \mathrm{H}-4,140-150$ & 32.5 & 10.0 & 3.3 & 1.18 & 41.1 & 3.4 & 0.19 & 0.27 & 0.07 & 0.05 & 37.74 & 98.81 \\
\hline $135-834 \mathrm{~A}-5 \mathrm{H}-4,140-150$ & 42.0 & 20.0 & 5.1 & 1.32 & 31.4 & 6.2 & 0.25 & 0.35 & 0.46 & 0.09 & 32.14 & 100.66 \\
\hline $135-834 \mathrm{~A}-6 \mathrm{H}-4,140-150$ & 51.0 & 26.7 & 7.7 & 1.88 & 26.5 & 6.6 & 0.43 & 0.38 & 1.40 & 0.17 & 25.36 & 99.02 \\
\hline $135-834 \mathrm{~A}-7 \mathrm{H}-4,130-132$ & 60.9 & 16.7 & 4.8 & 1.65 & 30.4 & 8.8 & 0.29 & 0.49 & 0.45 & 0.05 & 30.11 & 99.77 \\
\hline $135-834 \mathrm{~A}-8 \mathrm{H}-5,78-80$ & 71.4 & 17.9 & 5.3 & 1.55 & 28.7 & 10.3 & 0.28 & 0.66 & 0.58 & 0.11 & 29.66 & 100.56 \\
\hline $135-834 \mathrm{~A}-9 \mathrm{H}-4,140-150$ & 80.0 & 29.0 & 7.5 & 1.90 & 21.3 & 9.6 & 0.37 & 0.61 & 2.18 & 0.18 & 24.85 & 100.32 \\
\hline 135-834A-10X-2, 89-91 & 86 & 43.6 & 9.0 & 1.29 & 17.1 & 4.7 & 0.37 & 0.29 & 3.62 & 0.90 & 18.61 & 100.53 \\
\hline $135-834 A-11 X-2,71-73$ & 95.4 & 62.9 & 11.2 & 1.28 & 3.8 & 4.2 & 0.48 & 0.20 & 6.53 & 1.91 & 7.02 & 100.11 \\
\hline $135-834 A-12 X-1,140-150$ & 104.3 & 25.8 & 7.2 & 1.95 & 23.1 & 9.2 & 0.40 & 0.56 & 1.52 & 0.14 & 26.07 & 98.65 \\
\hline $135-834 \mathrm{~A}-16 \mathrm{X}-1,140-150$ & 133.3 & 59.0 & 13.2 & 2.56 & 8.0 & 6.2 & 0.51 & 0.22 & 5.78 & 0.98 & 4.28 & 101.16 \\
\hline $\begin{array}{c}\text { Core, Section } \\
\text { Interval }(\mathrm{cm})\end{array}$ & $\begin{array}{l}\text { Depth } \\
\text { (mbsf) }\end{array}$ & $\begin{array}{l}\mathrm{SiO} 2 \\
(\mathrm{Wt} \%)\end{array}$ & $\begin{array}{l}\mathrm{Al} 2 \mathrm{O3} \\
(\mathrm{Wt} \%)\end{array}$ & $\begin{array}{l}\mathrm{MgO} \\
(\mathrm{Wt} \%)\end{array}$ & $\begin{array}{l}\mathrm{CaO} \\
(\mathrm{Wt} \%)\end{array}$ & $\begin{array}{l}\mathrm{Fe} 2 \mathrm{O} 3 \\
\text { (Wt \%) }\end{array}$ & $\begin{array}{c}\mathrm{TiO} 2 \\
(\mathrm{Wt} \%)\end{array}$ & $\begin{array}{l}\mathrm{P} 205 \\
(\mathrm{Wt} \%)\end{array}$ & $\begin{array}{l}\mathrm{Na} 2 \mathrm{O} \\
(\mathrm{Wt} \%)\end{array}$ & $\begin{array}{l}\mathrm{K} 2 \mathrm{O} \\
(\mathrm{Wt} \%)\end{array}$ & \begin{tabular}{|} 
weight lost \\
at $1000^{\circ} \mathrm{C}$
\end{tabular} & Total \\
\hline $135-835 \mathrm{~A}-1 \mathrm{H}-4,140-150$ & 6 & .17 .7 & 4.4 & 1.30 & 35.1 & 4.9 & 0.21 & 0.33 & 0.18 & 0.05 & 33.10 & 99.17 \\
\hline $135-835 \mathrm{~A}-2 \mathrm{H}-4,140-150$ & 15.5 & 14.9 & 4.0 & 1.16 & 37.8 & 4.3 & 0.22 & 0.31 & 0.05 & 0.05 & & 99.36 \\
\hline $135-835 \mathrm{~A}-3 \mathrm{H}-4,140-150$ & 25.0 & 15.1 & 4.1 & 1.34 & 35.7 & 5.1 & 0.23 & 0.32 & 0.46 & 0.07 & 34.71 & 99.04 \\
\hline $135-835 \mathrm{~A}-4 \mathrm{H}-4,140-150$ & 34.0 & 16.4 & 4.2 & 1.19 & 36.5 & 4.8 & 0.21 & 0.28 & 0.49 & 0.07 & 33.95 & 99.59 \\
\hline $135-835 \mathrm{~A}-5 \mathrm{H}-5,90-92$ & 44.9 & 15.1 & 4.1 & 1.28 & 36.9 & 4.8 & 0.22 & 0.34 & 0.40 & 0.06 & 33.97 & 99.09 \\
\hline $135-835 \mathrm{~A}-6 \mathrm{H}-4,140-150$ & 53.5 & 15.4 & 4.1 & 1.19 & 36.3 & 4.6 & 0.22 & 0.30 & 0.45 & 0.07 & 34.36 & 98.72 \\
\hline $135-835 \mathrm{~A}-8 \mathrm{H}-4,140-150$ & 72.5 & 7.6 & 2.5 & 0.91 & 42.7 & 3.5 & 0.17 & 0.30 & 0.08 & 0.05 & 39.36 & 99.04 \\
\hline $135-835 \mathrm{~A}-9 \mathrm{H}-3,70-72$ & 79.7 & 12.1 & 3.9 & 1.36 & 38.6 & 4.8 & 0.24 & 0.27 & 0.14 & 0.05 & 36.66 & 99.32 \\
\hline $135-835 \mathrm{~A}-10 \mathrm{H}-4,140-150$ & 91.5 & 13.8 & 4.1 & 1.37 & 34.9 & 5.6 & 0.27 & 0.28 & 0.26 & 0.05 & 35.21 & 98.90 \\
\hline $135-835 \mathrm{~A}-12 \mathrm{H}-4,140-150$ & 110.5 & 13.1 & 3.9 & 1.30 & 34.8 & 7.1 & 0.22 & 0.36 & 0.25 & 0.05 & 35.03 & 99.81 \\
\hline $135-835 \mathrm{~A}-13 \mathrm{H}-4,110-112$ & 119.6 & 9.4 & 2.9 & 1.01 & 42.3 & 3.0 & 0.18 & 0.24 & 0.29 & 0.06 & 38.55 & 99.07 \\
\hline $135-835 \mathrm{~A}-14 \mathrm{H}-4,140-150$ & 129.5 & 12.7 & 3.5 & 1.22 & 36.2 & 6.9 & 0.20 & 0.41 & 0.35 & 0.05 & 33.07 & 100.55 \\
\hline $135-835 \mathrm{~A}-15 \mathrm{H}-4,121-123$ & 138.7 & 28.8 & 7.8 & 2.00 & 21.7 & 8.7 & 0.49 & 0.49 & 2.15 & 0.27 & 23.34 & 98.96 \\
\hline $135-835 A-16 X-6,140-150$ & 148.5 & 23.1 & 6.5 & 1.64 & 18.0 & 12.8 & 0.38 & 0.99 & 2.42 & 0.38 & 23.56 & 98.47 \\
\hline
\end{tabular}

\begin{tabular}{|c|c|c|c|c|c|c|c|c|c|c|c|c|}
\hline $\begin{array}{l}\text { Core, Section } \\
\text { Interval (cm) }\end{array}$ & $\begin{array}{l}\text { Depth } \\
\text { (mbsi) }\end{array}$ & $\begin{array}{l}\mathrm{SiO} 2 \\
(\mathrm{Wt} \%)\end{array}$ & & $\begin{array}{c}\mathrm{MgO} \\
(\mathrm{Wt} \%)\end{array}$ & $\begin{array}{l}\mathrm{CaO} \\
\left(\mathrm{W}_{t} \%\right)\end{array}$ & & $\begin{array}{l}\mathrm{TiO} 2 \\
\left(\mathrm{~W}_{t} \%\right)\end{array}$ & & & $\begin{array}{l}\mathrm{K} 2 \mathrm{O} \\
(\mathrm{Wt} \%)\end{array}$ & $\begin{array}{r}\text { weight lost } \\
\text { at } 1000^{\circ} \mathrm{C}\end{array}$ & Total \\
\hline & 7.2 & & 6.7 & & & 7.0 & & & & 0.27 & & \\
\hline & & & & 4 & 2 & 3. & .4 & .4 & 81 & .23 & 24.42 & \\
\hline
\end{tabular}

\begin{tabular}{|c|c|c|c|c|c|c|c|c|c|c|c|c|}
\hline $\begin{array}{c}\text { Core, Section } \\
\text { Interval (cm) }\end{array}$ & $\begin{array}{l}\text { Depth } \\
\text { (mbsf }\end{array}$ & $\begin{array}{l}\mathrm{SiO} 2 \\
(\mathrm{~W} t \%)\end{array}$ & $\begin{array}{l}\mathrm{Al} 2 \mathrm{O3} \\
(\mathrm{Wt} \%)\end{array}$ & $\begin{array}{l}\mathrm{MgO} \\
\left(\mathrm{W}^{\circ} \%\right)\end{array}$ & $\begin{array}{l}\mathrm{CaO} \\
(\mathrm{W} t \%)\end{array}$ & $\begin{array}{l}\mathrm{Fe} 2 \mathrm{O3} \\
(\mathrm{Wt} \%)\end{array}$ & $\begin{array}{l}\mathrm{TIO} 2 \\
(\mathrm{Wt} \%)\end{array}$ & $\begin{array}{l}\mathrm{P} 205 \\
\text { (Wt \%) }\end{array}$ & $\begin{array}{l}\mathrm{Na2O} \\
(\mathrm{W} t \%\rangle\end{array}$ & $\begin{array}{l}\mathrm{K} 2 \mathrm{O} \\
(\mathrm{Wt} \%)\end{array}$ & $\begin{array}{l}\text { weight lost } \\
1000^{\circ} \mathrm{C}\end{array}$ & Total \\
\hline $135-837 \mathrm{~A}-1 \mathrm{H}-3,140-150$ & 4.5 & 28.8 & 6.9 & 1.80 & 26.5 & 6.7 & 0.30 & 0.29 & 1.00 & 0.16 & 26.67 & 110.75 \\
\hline $135-837 \mathrm{~A}-2 \mathrm{H}-4,140-150$ & 14.0 & 17.2 & 4.5 & 1.32 & 35.1 & 5.7 & 0.22 & 0.31 & 0.07 & 0.05 & 33.60 & 99.97 \\
\hline $135-837 \mathrm{~A}-3 \mathrm{H}-4,140-150$ & 23.5 & 17.0 & 4.1 & 1.24 & 34.8 & 5.0 & 0.24 & 0.30 & 0.14 & 0.05 & 34.11 & 99.36 \\
\hline $135-837 \mathrm{~A}-4 \mathrm{H}-4,140-150$ & 31.5 & 64.3 & 12.1 & 1.09 & 4.2 & 3.6 & 0.45 & 0.21 & 5.34 & 1.38 & 5.56 & 98.39 \\
\hline $135-837 \mathrm{~A}-5 \mathrm{H}-4,130-132$ & 42.3 & 15.0 & 4.0 & 1.26 & 36.3 & 5.0 & 0.24 & 0.26 & 0.07 & 0.05 & 34.62 & 99.16 \\
\hline $135-837 \mathrm{~A}-6 \mathrm{H}-4,140-150$ & 53.5 & 13.4 & 4.2 & 1.50 & 37.7 & 5.8 & 0.25 & 0.31 & 0.05 & 0.05 & 35.02 & 100.38 \\
\hline $135-837 \mathrm{~A}-7 \mathrm{H}-1,18-20$ & 55.7 & 16.3 & 4.6 & 1.53 & 37.6 & 5.1 & 0.26 & 0.30 & 0.06 & 0.05 & 34.14 & 98.74 \\
\hline $135-837 \mathrm{~A}-8 \mathrm{H}-4,140-150$ & 71.0 & 22.9 & 6.0 & 1.69 & 29.9 & 6.4 & 0.34 & 0.29 & 0.64 & 0.12 & 31.55 & 100.63 \\
\hline $135-837 \mathrm{~A}-9 \mathrm{H}-5,78-80$ & 81.3 & 63.6 & 10.7 & 1.33 & 4.0 & 5.4 & 0.44 & 0.15 & 5.47 & 1.04 & 5.70 & 98.11 \\
\hline
\end{tabular}

\begin{tabular}{|c|c|c|c|c|c|c|c|c|c|c|c|c|}
\hline $\begin{array}{l}\text { Core, Section } \\
\text { Interval }(\mathrm{cm})\end{array}$ & $\begin{array}{l}\text { Depth } \\
\text { (mbsf) }\end{array}$ & $\begin{array}{l}\mathrm{SiO} 2 \\
(\mathrm{Wt} \%)\end{array}$ & $\begin{array}{l}\mathrm{A} 12 \mathrm{O} 3 \\
\text { (Wt \%) }\end{array}$ & $\begin{array}{l}\mathrm{MgO} \\
(\mathrm{W}(\%)\end{array}$ & $\begin{array}{l}\mathrm{CaO} \\
(\mathrm{Wt} \%)\end{array}$ & $\begin{array}{l}\mathrm{Fe} 2 \mathrm{O3} \\
(\mathrm{Wt} \%)\end{array}$ & $\begin{array}{r}\mathrm{TiO} 2 \\
(\mathrm{~W}+\%)\end{array}$ & $\begin{array}{l}\text { P2O5 } \\
(W t \%)\end{array}$ & $\begin{array}{l}\mathrm{Na} 2 \mathrm{O} \\
(\mathrm{W}+\%) \\
\end{array}$ & $\begin{array}{l}\mathrm{K} 2 \mathrm{O} \\
(\mathrm{W}) \%)\end{array}$ & $\begin{array}{r}\text { weight lost } \\
\text { at } 1000^{\circ} \mathrm{C} \\
\end{array}$ & Total \\
\hline $135-838 \mathrm{~A}-1 \mathrm{H}-1,50-52$ & 0.51 & 36.2 & 9.2 & 2.70 & 20.8 & 8.5 & 0.40 & 0.26 & 2.20 & 0.21 & & 101.15 \\
\hline & & & 3.7 & & & & & & & & & \\
\hline & & & 6. & & & & & & & & & \\
\hline & & & 6. & & 30 & 6 & & & & & & \\
\hline & & & 13.1 & & & & & & & & & 0.15 \\
\hline & 66 & 31 & 8.5 & & 23.5 & 6.7 & & & & 0.2 & 22.88 & 99.44 \\
\hline $135-8$ & & & 13. & & & & & & & & & \\
\hline 135-838A-11 H-5, 94-96 & 96.2 & 60.2 & 13.2 & 2.56 & 5.9 & 8.2 & 0.74 & 0.19 & 4.49 & 0.62 & 4.01 & 100.29 \\
\hline
\end{tabular}

\begin{tabular}{|c|c|c|c|c|c|c|c|c|c|c|c|c|}
\hline $\begin{array}{c}\text { Core, Section } \\
\text { Interval (cm) } \\
\end{array}$ & $\begin{array}{l}\text { Depth } \\
\text { (mbsf) }\end{array}$ & $\begin{array}{c}\mathrm{SiO} 2 \\
\left(\mathrm{~W}_{\dagger} \%\right)\end{array}$ & $\begin{array}{l}\mathrm{Al} 2 \mathrm{O} 3 \\
(\mathrm{~W}: \%)\end{array}$ & $\begin{array}{l}\mathrm{MgO} \\
(\mathrm{Wt} \%)\end{array}$ & $\begin{array}{l}\mathrm{CaO} \\
(\mathrm{Wt} \%)\end{array}$ & $\begin{array}{l}\mathrm{Fe} 2 \mathrm{O} 3 \\
\left(\mathrm{~W}_{\mathrm{t}} \%\right)\end{array}$ & $\begin{array}{c}\mathrm{TiO2} \\
\left(W_{t} \%\right)\end{array}$ & $\begin{array}{l}\mathrm{P} 2 \mathrm{O5} \\
(\mathrm{Wt} \%)\end{array}$ & $\begin{array}{l}\mathrm{Na} 2 \mathrm{O} \\
(\mathrm{Wt} \%)\end{array}$ & $\begin{array}{l}\mathrm{K} 2 \mathrm{O} \\
(\mathrm{W} t \%)\end{array}$ & $\begin{array}{l}\text { weight lost } \\
\text { at } 1000^{\circ} \mathrm{C}\end{array}$ & Total \\
\hline $135-839 \mathrm{~A}-1 \mathrm{H}-2,140-150$ & 3.0 & 47.2 & 10.7 & 2.38 & 12.4 & 8.3 & 0.45 & 0.26 & 3.56 & 0.68 & 10.89 & 98.23 \\
\hline $39 \mathrm{~A}-2 \mathrm{H}-4,140-150$ & 10.5 & 20.1 & 5.4 & 1.63 & 30.5 & 7.1 & 0.30 & 0.27 & 0.40 & 0.05 & 31.4 & 99.10 \\
\hline $135-839 \mathrm{~A}-3 \mathrm{H}-4,140-150$ & 20 & 65 & 10.7 & 0.78 & 4.0 & 3.3 & 0.33 & 0.15 & 4.77 & 1.13 & & 8.33 \\
\hline $135-\varepsilon$ & & & 7.0 & & 25.6 & 6.4 & & 0.29 & 1.20 & 0.21 & & 100.71 \\
\hline 135 & & & & & 28.0 & 7.7 & 0.37 & 0.28 & & 0.2 & & 100.90 \\
\hline & & 66. & 11.7 & 1.2 & 4.5 & 3.5 & 0.4 & 0.1 & 4.8 & 1.31 & & 100.78 \\
\hline 135 & & & & & 4.1 & 3. & & & & 1.3 & & 101.00 \\
\hline $135-\varepsilon$ & & & & & 4.2 & 3. & 0.4 & & 4.6 & 1.27 & 6.7 & 100.85 \\
\hline & & & 11 & & 4.8 & 3. & & & & & & \\
\hline $135-8$ & & & & & 18.2 & 5. & & & & 0.4 & & 98.67 \\
\hline & & & 10. & 1.3 & 5.6 & 3. & & & 4 & 1.8 & & 100.83 \\
\hline $135-8$ & & & & & & & & & & 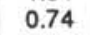 & & 99.93 \\
\hline & & 46 & 13.3 & 5.46 & 11.2 & 10.0 & 0.5 & 0.3 & 2.7 & 1.09 & & 100.90 \\
\hline $135-839 A-21 X-c c, 1$ & 186 & 53.2 & 15.2 & 4.51 & 9.4 & 9.5 & 0.71 & 0.21 & 3.06 & 0.72 & 4.45 & 101.19 \\
\hline
\end{tabular}

Notes: Total calculation includes $\mathrm{Ba}, \mathrm{Sr}$, and $\mathrm{Mn}$, calculated as oxides, from Table 2 . 
Table 2. Minor element composition of bulk sediments, Holes 834A-839A.

\begin{tabular}{|c|c|c|c|c|c|c|c|c|c|c|c|c|c|c|}
\hline $\begin{array}{l}\text { Core, } \\
\text { Interval }\end{array}$ & $\begin{array}{l}\text { Section } \\
\text { (cm) }\end{array}$ & $\begin{array}{l}\text { Depth } \\
\text { (mbsf) }\end{array}$ & $\begin{array}{c}\text { Mn } \\
(g / \mathrm{kg})\end{array}$ & $\begin{array}{c}\mathrm{Sr} \\
(\mathrm{mg} / \mathrm{kg})\end{array}$ & $\begin{array}{c}\mathrm{Ba} \\
(\mathrm{mg} / \mathrm{kg})\end{array}$ & $\underset{(\mathrm{mg} / \mathrm{kg})}{V}$ & $\begin{array}{c}\mathrm{Cr} \\
(\mathrm{mg} / \mathrm{kg})\end{array}$ & $\begin{array}{c}\mathrm{Ni} \\
(\mathrm{mg} / \mathrm{kg})\end{array}$ & $\begin{array}{c}\mathrm{Co} \\
(\mathrm{mg} / \mathrm{kg})\end{array}$ & $\begin{array}{c}\mathrm{Zn} \\
(\mathrm{mg} / \mathrm{kg})\end{array}$ & $\begin{array}{c}\mathrm{Cu} \\
(\mathrm{mg} / \mathrm{kg})\end{array}$ & $\begin{array}{c}\mathrm{Zr} \\
(\mathrm{mg} / \mathrm{kg})\end{array}$ & $\begin{array}{c}Y \\
(\mathrm{mg} / \mathrm{kg})\end{array}$ & $\begin{array}{c}\mathrm{Sc} \\
(\mathrm{mg} / \mathrm{kg})\end{array}$ \\
\hline $135-834 \mathrm{~A}-1 \mathrm{H}-4$ & $140-150$ & 5.9 & 1.40 & 3155 & 113 & 20.3 & 13.0 & 38.0 & 7.4 & 14.5 & 20.1 & 7.7 & 9.6 & 2.0 \\
\hline $135-834 \mathrm{~A}-2 \mathrm{H}-4$ & $140-150$ & 13.5 & 9.99 & 1407 & 517 & 109.0 & 16.0 & 58.2 & 22.8 & 46.1 & 37.0 & 24.8 & 24.6 & 8.8 \\
\hline $135-834 \mathrm{~A}-3 \mathrm{H}-4$, & $140-150$ & 23.0 & 9.48 & 1109 & 460 & 73.8 & 18.8 & 59.0 & 20.1 & 41.0 & 26.4 & 37.4 & 27.8 & 6.6 \\
\hline $135-834 \mathrm{~A}-4 \mathrm{H}-4$ & $140-150$ & 32.5 & 8.71 & 1348 & 311 & 80 & 12.5 & 59.7 & 19.3 & 33.7 & 28.6 & 27.0 & 22.5 & 6.7 \\
\hline $135-834 \mathrm{~A}-5 \mathrm{H}-4$ & $140-150$ & 42.0 & 21.98 & 1374 & 537 & 140 & 17.6 & 150.0 & 25.0 & 128.0 & 65.4 & 43.2 & 33.6 & 7.9 \\
\hline $135-834 \mathrm{~A}-6 \mathrm{H}-4$, & $140-150$ & 51.0 & 10.75 & 1163 & 442 & 143 & 49.4 & 72.9 & 27.2 & 118.0 & 74.9 & 54.3 & 26.2 & 11.6 \\
\hline $135-834 \mathrm{~A}-7 \mathrm{H}-4$, & $130-132$ & 60.9 & 41.28 & 1491 & 634 & 292 & 50.8 & 193.0 & 35.5 & 184.0 & 121.0 & 43.7 & 34.7 & 10.2 \\
\hline $135-834 \mathrm{~A}-8 \mathrm{H}-5$, & $78-80$ & 71.4 & 38.48 & 1284 & 525 & 334 & 29.2 & 161.0 & 34.8 & 179.0 & 182.0 & 44.8 & 33.3 & 9.2 \\
\hline $135-834 \mathrm{~A}-9 \mathrm{H}-4$ & $140-150$ & 80.0 & 18.52 & 1040 & 465 & 134 & 30.5 & 68.2 & 37.7 & 159.0 & 69.1 & 58.9 & 31.5 & 11.7 \\
\hline $135-834 A-10 X-2$, & $89-91$ & 86.0 & 6.24 & 750 & 267 & 82.8 & 14.5 & 42.7 & 16.7 & 87.6 & 38.3 & 67.4 & 30.9 & 11.1 \\
\hline $135-834 A-11 X-2$ & $71-73$ & 95.4 & 3.93 & 170 & 174 & 43.4 & 13.5 & 30.9 & 14.0 & 58.0 & 60.8 & 131.0 & 42.6 & 14.4 \\
\hline $135-834 \mathrm{~A}-12 \mathrm{X}-2$ & $140-150$ & 104.3 & 17.36 & 972 & 473 & 277.0 & 36.2 & 71.2 & 27.0 & 155.0 & 105.0 & 57.2 & 30.4 & 11.6 \\
\hline $135-834 \mathrm{~A}-16 \mathrm{X}-1$ & $140-150$ & 133.3 & 2.84 & 320 & 558 & 129.0 & 16.3 & 34.5 & 22.1 & 91.7 & 56.4 & 89.3 & 34.2 & 20.6 \\
\hline
\end{tabular}

\begin{tabular}{|c|c|c|c|c|c|c|c|c|c|c|c|c|c|c|}
\hline $\begin{array}{r}\text { Core, } \\
\text { Interval }\end{array}$ & $\begin{array}{l}\text { Section } \\
\text { (cm) }\end{array}$ & $\begin{array}{l}\text { Depth } \\
\text { (mbsf) }\end{array}$ & $\begin{array}{c}\text { Mn } \\
(g / \mathrm{kg})\end{array}$ & $\begin{array}{c}\mathrm{Sr} \\
(\mathrm{mg} / \mathrm{kg})\end{array}$ & $\begin{array}{c}\mathrm{Ba} \\
(\mathrm{mg} / \mathrm{kg})\end{array}$ & $\begin{array}{c}V \\
(\mathrm{mg} / \mathrm{kg})\end{array}$ & $\begin{array}{c}\mathrm{Cr} \\
(\mathrm{mg} / \mathrm{kg})\end{array}$ & $\begin{array}{c}\mathrm{Ni} \\
(\mathrm{mg} / \mathrm{kg})\end{array}$ & $\begin{array}{c}\mathrm{Co} \\
(\mathrm{mg} / \mathrm{kg})\end{array}$ & $\begin{array}{c}\mathrm{Zn} \\
(\mathrm{mg} / \mathrm{kg})\end{array}$ & $\begin{array}{c}\mathrm{Cu} \\
(\mathrm{mg} / \mathrm{kg})\end{array}$ & $\begin{array}{c}\mathrm{Zr} \\
(\mathrm{mg} / \mathrm{kg})\end{array}$ & $\begin{array}{c}Y \\
(\mathrm{mg} / \mathrm{kg})\end{array}$ & $\begin{array}{c}S c \\
(\mathrm{mg} / \mathrm{kg})\end{array}$ \\
\hline 135-835A-1म-4, & $140-150$ & 6.0 & 11.38 & 1197 & 518 & 130.0 & 18.3 & 71.2 & 24.4 & 95.1 & 24.8 & 32.1 & 27.6 & 10.6 \\
\hline $135-835 \mathrm{~A}-2 \mathrm{H}-4$. & $140-150$ & 15.5 & 10.26 & 1182 & 484 & 105.0 & 16.9 & 63.2 & 20.7 & 64.1 & 36.9 & 28.6 & 27.0 & 8.8 \\
\hline $135-835 \mathrm{~A}-3 \mathrm{H}-4$, & $140-150$ & 25.0 & 12.05 & 1229 & 492 & 116.0 & 6.7 & 71.4 & 26.6 & 66.4 & 98.7 & 24.7 & 25.9 & 8.6 \\
\hline $135-835 \mathrm{~A}-4 \mathrm{H}-4$ & $140-150$ & 34 & 9.26 & 1254 & 525 & 104.0 & 8.9 & 58.4 & 26.1 & 69.9 & 72.3 & 24.5 & 26.8 & 9.5 \\
\hline $135-835 \mathrm{~A}-5 \mathrm{H}-5$, & $90-92$ & 44.9 & 12.47 & 1321 & 590 & 113.0 & 10.1 & 67.9 & 31.7 & 75.8 & 86.2 & 27.8 & 26.9 & 10.0 \\
\hline $135-835 \mathrm{~A}-6 \mathrm{H}-4$. & $140-150$ & 53.5 & 10.40 & 1209 & 596 & 95.2 & 8.9 & 61.9 & 28.1 & 61.6 & 72.3 & 26.0 & 29.7 & 8.9 \\
\hline $135-835 \mathrm{~A}-8 \mathrm{H}-4$ & $140-150$ & 72.5 & 11.46 & 1405 & 529 & 76.0 & 6.2 & 75.9 & 27.5 & 60.7 & 72.5 & 21.3 & 26.9 & 5.2 \\
\hline $135-835 \mathrm{~A}-9 \mathrm{H}-3$ & $70-72$ & 79.7 & 7.60 & 1316 & 483 & 97.7 & 12.8 & 81.1 & 23.5 & 71.7 & 101.0 & 32.2 & 30.4 & 8.8 \\
\hline $135-835 \mathrm{~A}-10 \mathrm{H}-4$ & $140-150$ & 91.5 & 19.03 & 1347 & 567 & 125.0 & 12.6 & 150.0 & 38.0 & 94.9 & 104.0 & 32.7 & 32.5 & 8.2 \\
\hline $135-835 \mathrm{~A}-12 \mathrm{H}-4$, & $140-150$ & 110.5 & 25.58 & 1462 & 448 & 163.0 & 19.5 & 147.0 & 30.6 & 112.0 & 136.0 & 31.9 & 28.4 & 8.3 \\
\hline $135-835 \mathrm{~A}-13 \mathrm{H}-4$ & $110-112$ & 119.6 & 7.19 & 1154 & 198 & 67.6 & 20.4 & 37.9 & 16.4 & 52.3 & 35.3 & 22.3 & 20.5 & 6.3 \\
\hline $135-835 \mathrm{~A}-14 \mathrm{H}-4$ & $140-150$ & 129.5 & 41.43 & 1432 & 528 & 211.0 & 18.8 & 159.0 & 30.8 & 140.0 & 157.0 & 33.9 & 29.1 & 7.5 \\
\hline $135-835 A-15 H-4$ & $121-123$ & 138.7 & 21.69 & 1028 & 464 & 174.0 & 26.6 & 88.5 & 43.5 & 150.0 & 109.0 & 50.3 & 29.6 & 14.9 \\
\hline $135-835 A-16 X-6$ & $140-150$ & 148.5 & 61.10 & 1187 & 755 & 492.0 & 30.1 & 196.0 & 46.8 & 243.0 & 310.0 & 69.3 & 49.8 & 11.5 \\
\hline
\end{tabular}

\begin{tabular}{|c|c|c|c|c|c|c|c|c|c|c|c|c|c|}
\hline $\begin{array}{l}\text { Core, Section } \\
\text { Interval }(\mathrm{cm})\end{array}$ & $\begin{array}{l}\text { Depth } \\
\text { (mbsf) }\end{array}$ & $\begin{array}{c}\mathrm{Mn} \\
(g / \mathrm{kg})\end{array}$ & $\begin{array}{c}\mathrm{Sr} \\
(\mathrm{mg} / \mathrm{kg})\end{array}$ & $\begin{array}{c}\mathrm{Ba} \\
(\mathrm{mg} / \mathrm{kg})\end{array}$ & $\begin{array}{c}V \\
(\mathrm{mg} / \mathrm{kg})\end{array}$ & $\begin{array}{c}\mathrm{Cr} \\
(\mathrm{mg} / \mathrm{kg})\end{array}$ & $\begin{array}{c}\mathrm{Ni} \\
(\mathrm{mg} / \mathrm{kg})\end{array}$ & $\begin{array}{c}\mathrm{Co} \\
(\mathrm{mg} / \mathrm{kg})\end{array}$ & $\begin{array}{c}\mathrm{Zn} \\
(\mathrm{mg} / \mathrm{kg})\end{array}$ & $\begin{array}{c}\mathrm{Cu} \\
(\mathrm{mg} / \mathrm{kg})\end{array}$ & $\begin{array}{c}\mathrm{Zr} \\
(\mathrm{mg} / \mathrm{kg})\end{array}$ & $\begin{array}{c}Y \\
(\mathrm{mg} / \mathrm{kg})\end{array}$ & $\begin{array}{c}\mathrm{Sc} \\
(\mathrm{mg} / \mathrm{kg})\end{array}$ \\
\hline $135-836 \mathrm{~A}-2 \mathrm{H}-4,140-150$ & 7.2 & 12.52 & 1006 & 509 & 180.0 & 17.2 & 62.5 & 34.3 & 95.0 & 69.5 & 27.7 & 24.3 & 17.5 \\
\hline $\begin{array}{l}135-836 \mathrm{~A}-2 \mathrm{H}-5, \mid 140-150\end{array}$ & 16.7 & 12.87 & 869 & 544 & 200.0 & 21.6 & 96.4 & 40.4 & 112.0 & 78.5 & 39.1 & 32.2 & 17.6 \\
\hline
\end{tabular}

\begin{tabular}{|c|c|c|c|c|c|c|c|c|c|c|c|c|c|}
\hline $\begin{array}{l}\text { Core, Section } \\
\text { Interval }(\mathrm{cm})\end{array}$ & $\begin{array}{l}\text { Depth } \\
\text { (mbst) }\end{array}$ & $\begin{array}{c}\mathrm{Mn}_{(g / \mathrm{kg})} \\
(g)^{2}\end{array}$ & $\begin{array}{c}\mathrm{Sr} \\
(\mathrm{mg} / \mathrm{kg})\end{array}$ & $\begin{array}{c}\mathrm{Ba} \\
(\mathrm{mg} / \mathrm{kg})\end{array}$ & $\begin{array}{c}V \\
(\mathrm{mg} / \mathrm{kg})\end{array}$ & $\begin{array}{c}\mathrm{Cr} \\
(\mathrm{mg} / \mathrm{kg})\end{array}$ & $\begin{array}{c}\mathrm{Ni} \\
(\mathrm{mg} / \mathrm{kg})\end{array}$ & $\begin{array}{c}\text { Co } \\
(\mathrm{mg} / \mathrm{kg})\end{array}$ & $\begin{array}{c}\mathrm{Zn} \\
(\mathrm{mg} / \mathrm{kg})\end{array}$ & $\begin{array}{c}\mathrm{Cu} \\
(\mathrm{mg} / \mathrm{kg})\end{array}$ & $\begin{array}{c}\mathrm{Zr} \\
(\mathrm{mg} / \mathrm{kg})\end{array}$ & $\begin{array}{c}Y \\
(\mathrm{mg} / \mathrm{kg})\end{array}$ & $\begin{array}{c}\mathrm{Sc} \\
(\mathrm{mg} / \mathrm{kg})\end{array}$ \\
\hline $135-837 \mathrm{~A}-1 \mathrm{H}-3,140-150$ & 4.5 & 10.05 & 918 & 602 & 153.0 & 18.1 & 82.4 & 32.2 & 91.2 & 36.7 & 29.2 & 27.0 & 15.6 \\
\hline $135-837 \mathrm{~A}-2 \mathrm{H}-4,140-150$ & 14.0 & 11.94 & 1138 & 585 & 123.0 & 16.2 & 95.5 & 28.0 & 72.2 & 24.8 & & 28.6 & 10.2 \\
\hline $\begin{array}{l}135-837 \mathrm{~A}-3 \mathrm{H}-4,140-150 \\
\end{array}$ & 23.5 & 14.73 & 1232 & 632 & 118.0 & 23.8 & 145.0 & 32.7 & 67.5 & 24 & 35.1 & 37.0 & 8.8 \\
\hline 135-837A-4H-4, $140-150$ & 31.5 & 0.96 & 169 & 255 & 47.9 & 12.0 & 28.2 & 19.2 & 28.5 & 61.8 & 85.4 & 31.9 & 24.4 \\
\hline $135-837 \mathrm{~A}-5 \mathrm{H}-4,[130-132$ & 42.3 & 14.84 & 1187 & 579 & 116.0 & 22.9 & 167.0 & 34.1 & 28 & 21 & 31.9 & 34.2 & 8.7 \\
\hline \begin{tabular}{l|l}
$135-837 A-6 H-4$ & $140-150$
\end{tabular} & 53.5 & 11.51 & 1258 & 533 & 68.2 & 16.7 & 113.0 & 44.3 & 59.0 & 48.6 & 31.0 & 26.9 & 9.2 \\
\hline $135-837 \mathrm{~A}-7 \mathrm{H}-1,18-20$ & 55.7 & 11.88 & 1241 & 498 & 108.0 & 22.9 & 161.0 & 29.8 & 73 & 19 & 28 & 25.2 & 10.9 \\
\hline 135-837A-8H-4, $140-150$ & 71.0 & 4.48 & 1017 & 288 & 110.0 & 16.2 & 91.1 & 25.0 & 94. & 42 & 35.6 & 26.5 & 12.1 \\
\hline $135-837 \mathrm{~A}-9 \mathrm{H}-5,78-80$ & 81.3 & 1.38 & 146 & 147 & 46.5 & 6.7 & 37.5 & 18.3 & 56.9 & 65.9 & 36.7 & 34.2 & 18.8 \\
\hline
\end{tabular}

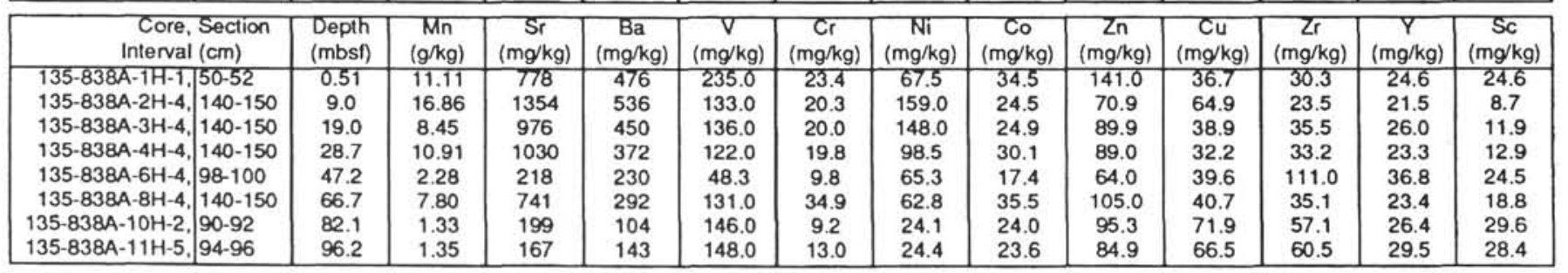

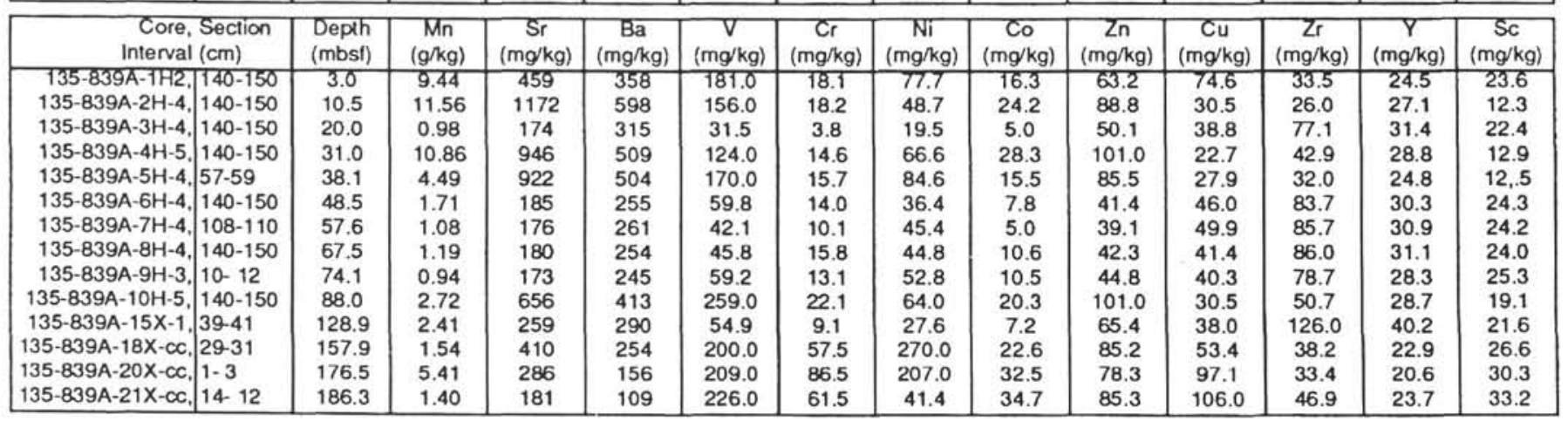


A Site 834A weight (\%)

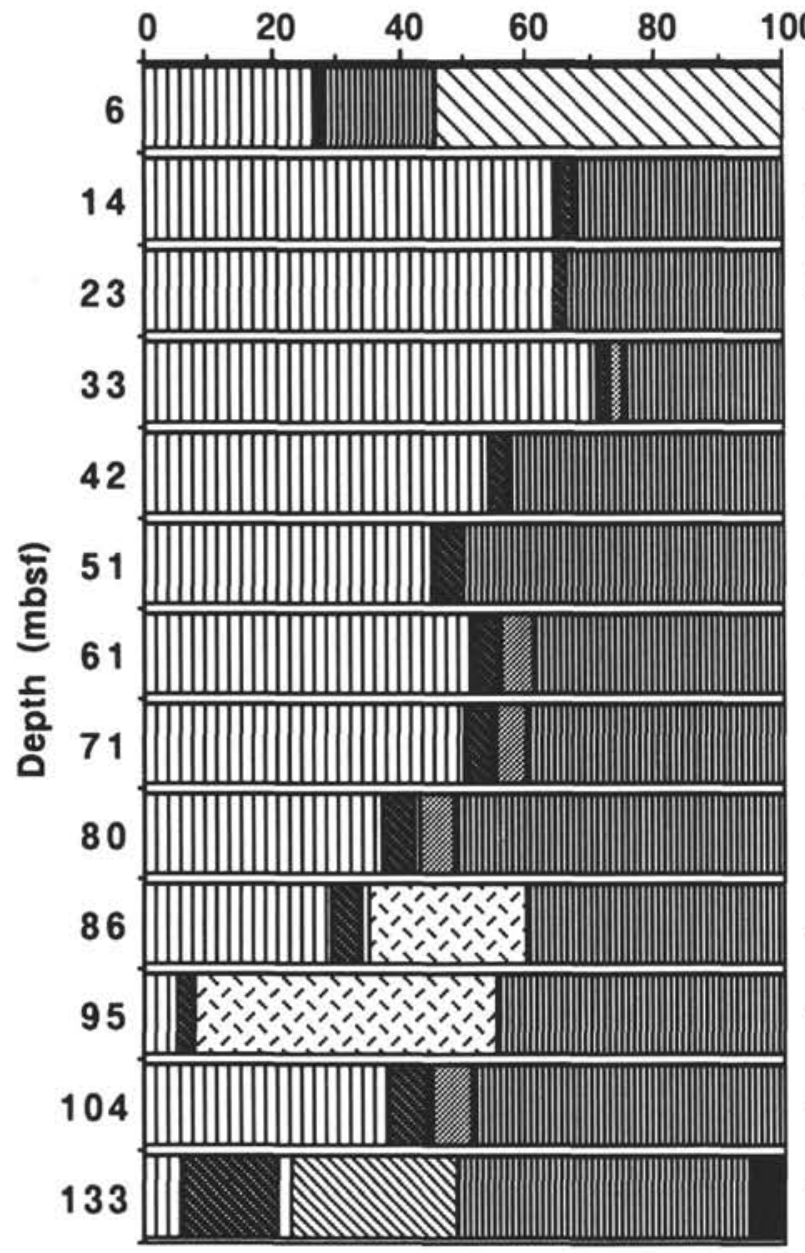

$1 \mathrm{H} 4$

$2 \mathrm{H} 4$ (1)

$3 \mathrm{H} 4$ (1)

$4 \mathrm{H} 4(1)$

$5 \mathrm{H} 4$

(1)

$6 \mathrm{H} 4(1)$

$7 \mathrm{H} 4(1)$

$8 \mathrm{H} 5$

(1)

$9 \mathrm{H} 4$

(2)

$10 \times 2$

(2)

$11 \times 2$

$12 \times 2$

(2)

$16 \times 1$
B Site 834A Weight (\%)

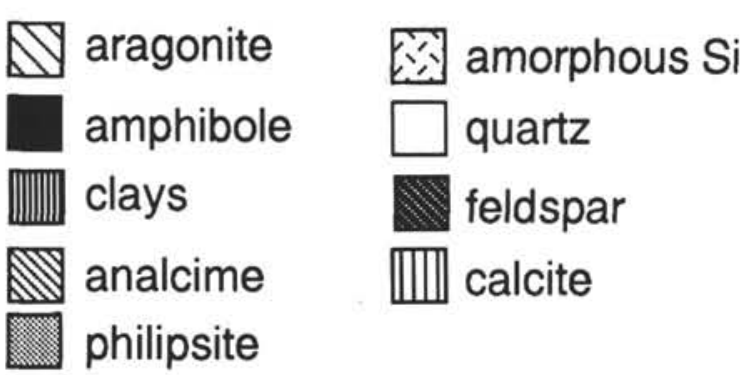

Figure 2. A. Cumulative histograms vs. depth of semiquantitative estimates of the solid phase abundances (\%) based on XRD diagrams, Hole 834A. B. Relative amounts of major chemical components in the bulk sediments (wt\%), Hole 834A. On the right side of the solid phase abundances is written the core and section number and the class number for each sample (class number in parentheses).

$135-834 \mathrm{~A}-1 \mathrm{H}-4,140-150 \mathrm{~cm}$, has a $\mathrm{Sr} / \mathrm{Ca}$ ratio $\left(9.24 \times 10^{-3}\right)$ that is 1.7 times that of the other samples $\left([5.3 \pm 1.0] \times 10^{-3}\right)$, reflecting the high content of aragonite in this sample. Aragonite precipitation selectively removes $\mathrm{Sr}$ (e.g., Graham et al., 1982). The correlation line $(\mathrm{Sr}=46.89 \mathrm{Ca}+70.72, r=0.961)$ is comparable to the line of biogenic calcite in marine sediments from 0 to 4 Ma presented by Baker et al. (1982). However, the difference of the slope between these two lines suggests a slight enrichment in Sr in the Lau sediments. For each class, samples having high $\mathrm{Mn} / \mathrm{Ca}$ ratios also yield high $\mathrm{Sr} / \mathrm{Ca}$ ratios (Fig. 12). Two groups of samples can be distinguished as a function of their Mn enrichment. Forty-five samples yield a $\mathrm{Mn} / \mathrm{Ca}$ ratio of $43.95 \times 10^{-3} \pm 0.73 \times 10^{-3}$ and a $\mathrm{Sr} / \mathrm{Ca}$ ratio of $4.97 \times 10^{-3} \pm$
$0.63 \times 10^{-3}$. Fourteen samples show a $\mathrm{Mn} / \mathrm{Ca}$ ratio of $128.6 \times 10^{-3} \pm$ $40.79 \times 10^{-3}$ and a Sr/Ca ratio of $6.29 \times 10^{-3} \pm 0.89 \times 10^{-3}$. The aragonite-rich sample $(135-834 \mathrm{~A}-1 \mathrm{H}-4,140-150 \mathrm{~cm})$ has a $\mathrm{Mn} / \mathrm{Ca}$ ratio of $4.43 \times 10^{-3}$ and a $\mathrm{Sr} / \mathrm{Ca}$ ratio of $9.24 \times 10^{-3}$. The samples most enriched in $\mathrm{Mn}$ and $\mathrm{Sr}$ with respect to $\mathrm{Ca}$ were collected predominantly in the deeper parts of Sites 834 and 835 . This suggests higher $\mathrm{Mn}$ and $\mathrm{Sr}$ accumulations in the sedimentary columns to the west of the CLSC, 3-4 m.y. ago. These $\mathrm{Sr}$ and $\mathrm{Mn}$ enrichments could be attributed to a secondary sink, such as manganese oxides, which were observed in the smear slides (Parson, Hawkins, Allan, et al., 1992) and also described in recent sediment from the Lau Basin (Riech, 1990). The Sr concentrations in manganese oxides from the Gala- 
A

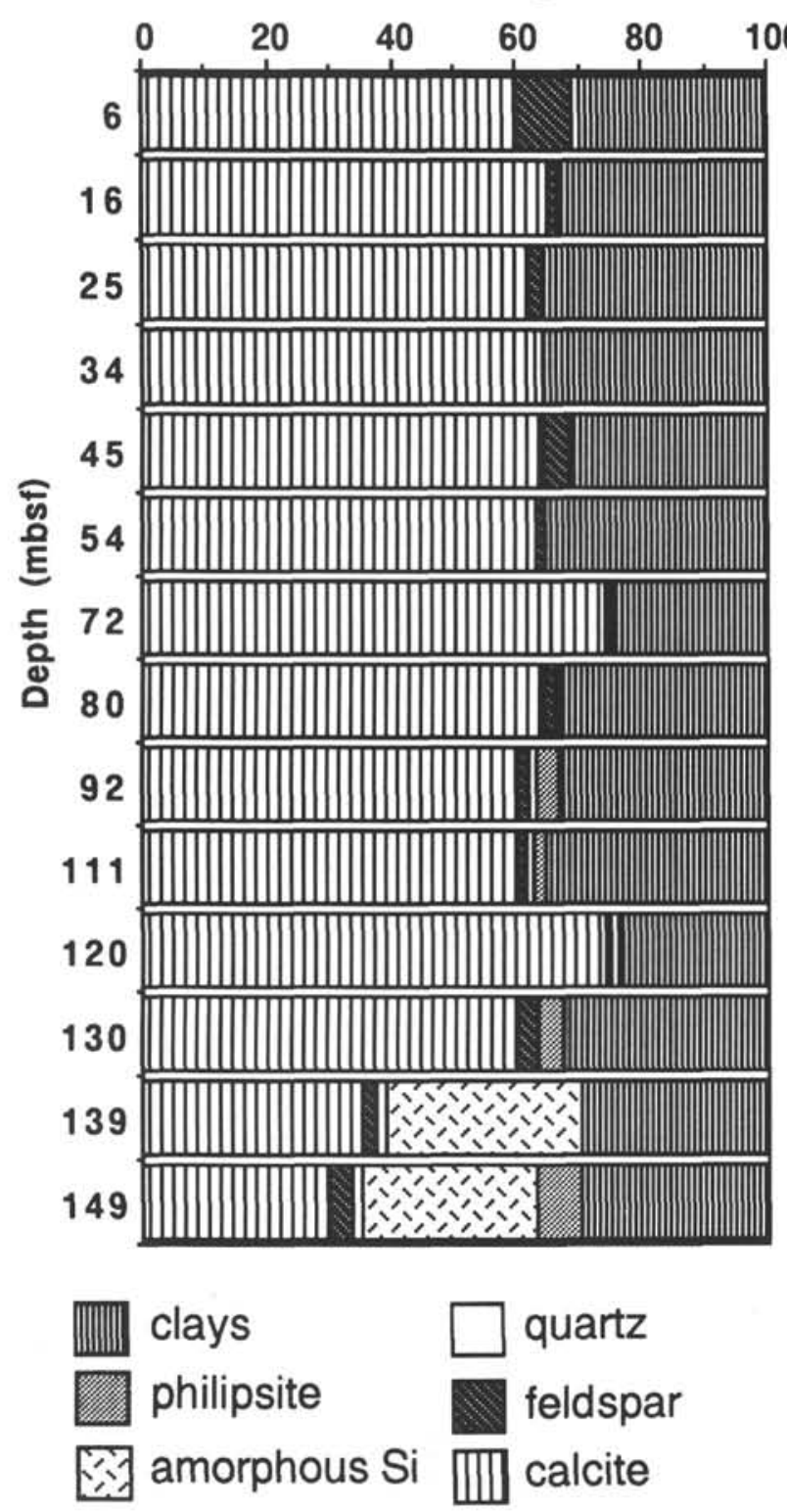

B

$1 \mathrm{H} 4(1)$

$2 \mathrm{H} 4$ (1)

$3 \mathrm{H} 4$ (1)

$4 \mathrm{H} 4$ (1)

$5 \mathrm{H} 5$ (1)

$6 \mathrm{H} 4$ (1)

$8 \mathrm{H} 4$ (1)

$9 \mathrm{H} 3$ (1)

$10 \mathrm{H} 4$ (1)

$12 \mathrm{H} 4$ (1)

$13 \mathrm{H} 4$ (1)

$14 \mathrm{H} 4$ (1)

$15 \mathrm{H} 4$ (2)

$16 \times 6$ (2)

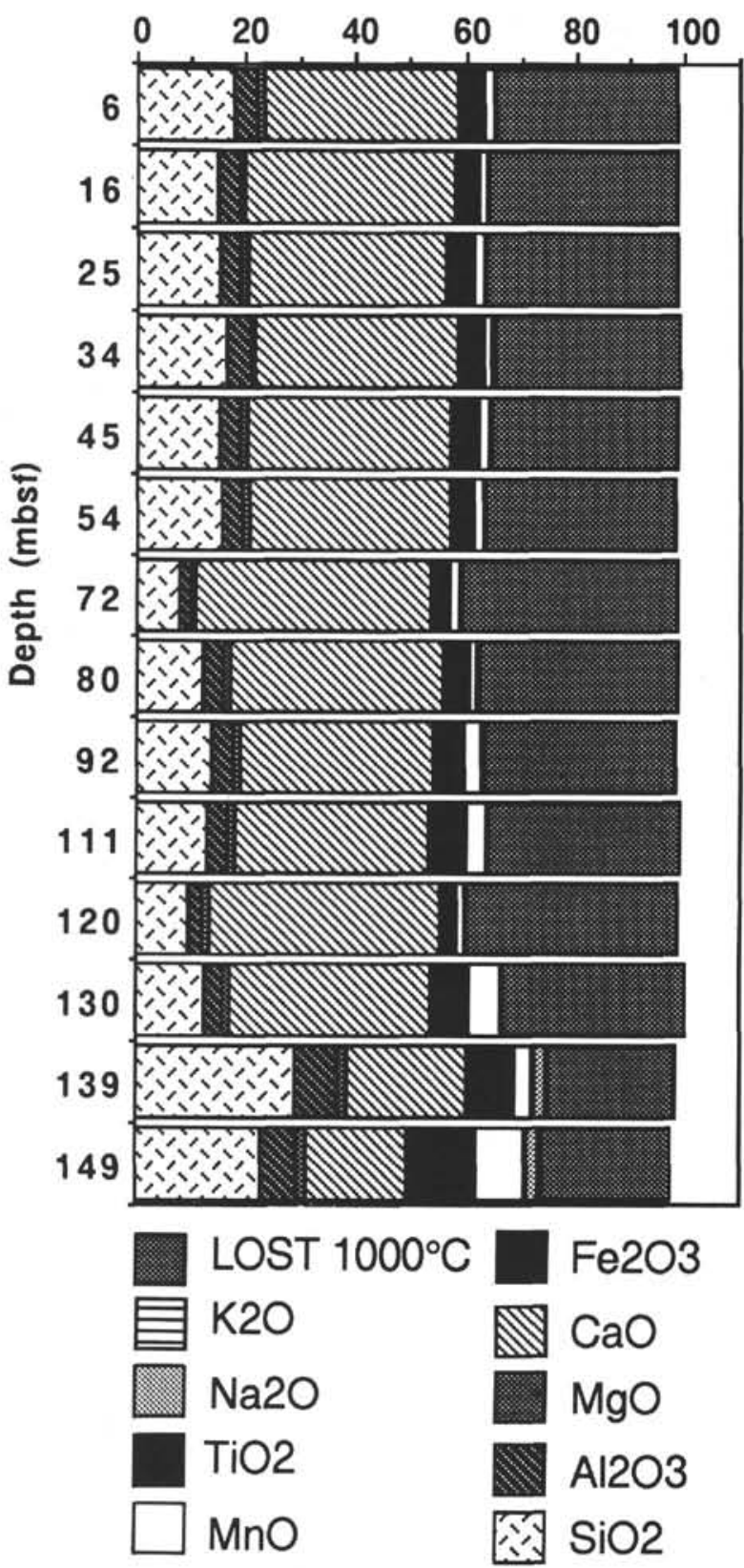

Figure 3. A. Cumulative histograms vs. depth of semiquantitative estimates of the solid phase abundances (\%) based on XRD diagrams, Hole 835A. B. Relative amounts of major chemical components in the bulk sediments (wt\%), Holes 835A. Parameters as in Figure 2.

pagos Spreading Center and from the Atlantis II Deep (Red Sea) can be as high as $1000 \mathrm{ppm}$ (Clauer et al., 1984; Blanc, 1987).

\section{Characterization of the Alteration Grade of the Volcanogenic Material}

The sediments of Sites 834 and 835 are mineralogically characterized by the occurrence of zeolites, which are authigenic minerals formed by halmyrolitic alteration of volcanic material. The distribution of zeolites in sediments depends on time and on physical and chemical parameters of the liquid and solid fractions present in the medium (Kastner and Stonecipher, 1978). Phillipsite is generally stable in various environments between $0^{\circ}$ and $80^{\circ} \mathrm{C}$. Occurrence of analcime in the Lau Basin sediments indicates a higher grade of al- teration than does the occurrence of phillipsite (von Rad et al., 1990). The presence of analcime in Sample 135-834A-16X-1, 140-150 cm, suggests temperatures higher than $100^{\circ} \mathrm{C}$ in the medium. This sample was collected in a thin sediment slab (Unit IV) interbedded between lower flows of vesicular basalt (Parson, Hawkins, Allan, et al., 1992). Hence, analcimization of this sample might have been produced by baking at the contact with basalt. Thus, the thermal gradients in Holes $834 \mathrm{~A}$ and $835 \mathrm{~A}$ were probably higher than those presently observed, which range from $1.5^{\circ}$ to $5^{\circ} \mathrm{C} / 100 \mathrm{~m}$ (Parson, Hawkins, Allan, et al., 1992). This result is in good agreement with the results obtained on the interstitial waters of the Lau Basin, which require that the diagenetically chemical and isotopic signatures of the pore waters are affected by a present downwelling flow of bottom seawater (Blanc et al., this volume). Zeolites were not detected by XRD in the other sites, thus 
A

Site 836A weight \%

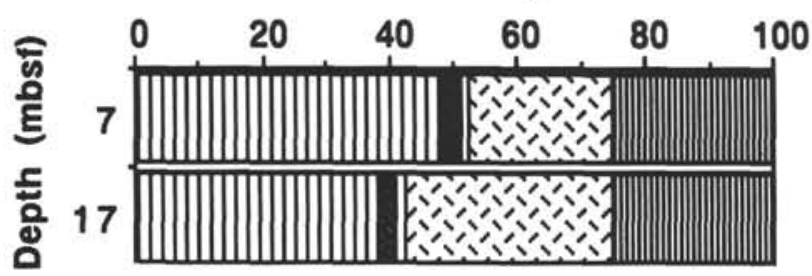

B

Site 836A weight (\%)

$2 \mathrm{H} 4$ (2)

$2 \mathrm{H} 5(2)$
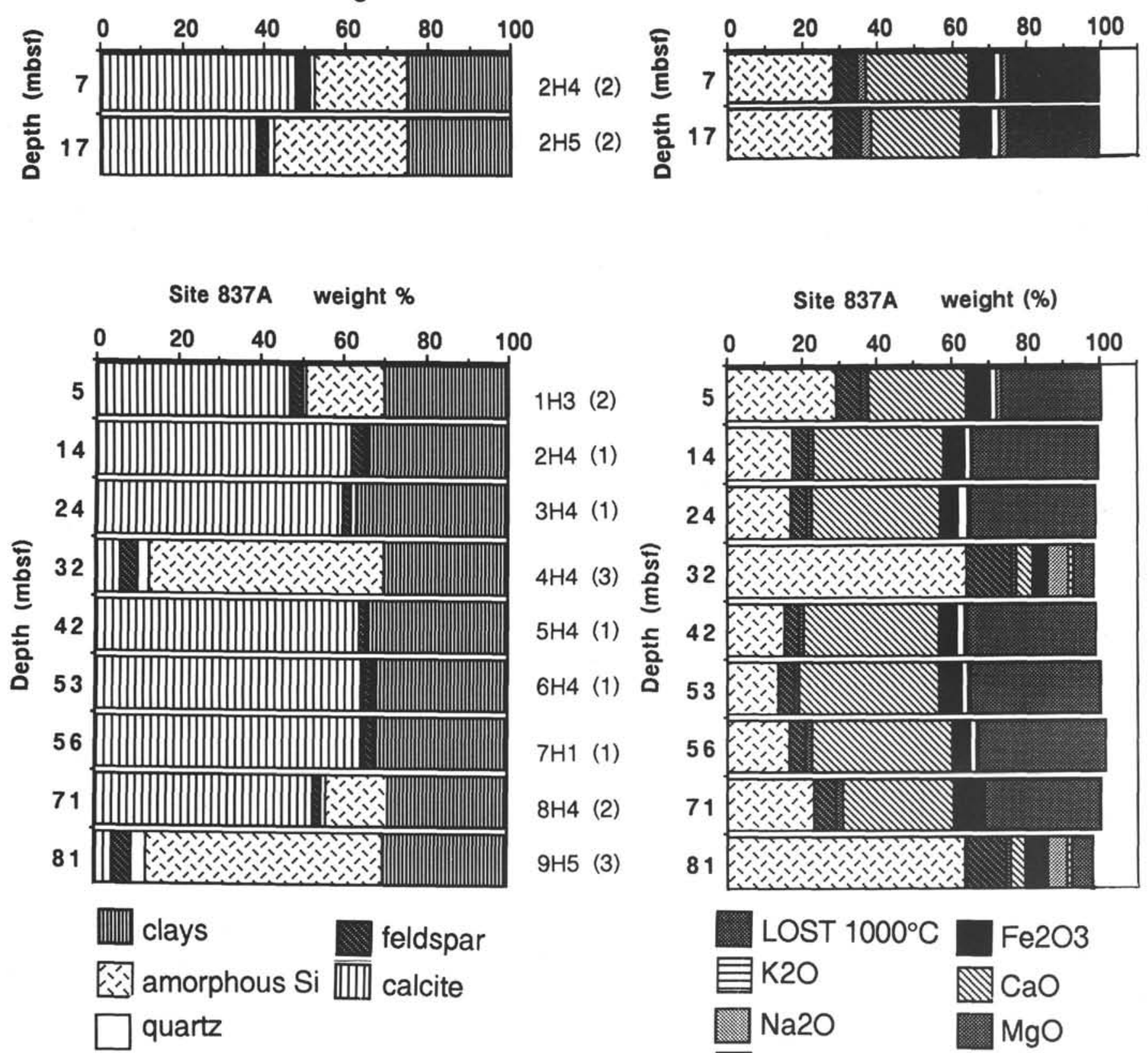

$1 \mathrm{H} 3$

(2)

$2 \mathrm{H} 4(1)$

$3 \mathrm{H} 4$ (1)

$4 \mathrm{H} 4$ (3) है

$5 \mathrm{H} 4$ (1)

$6 \mathrm{H} 4$ (1)

ㅎํㅇ

$7 \mathrm{H} 1(1)$

$8 \mathrm{H} 4$ (2)

$9 \mathrm{H} 5$ (3)

Site 837A weight (\%)
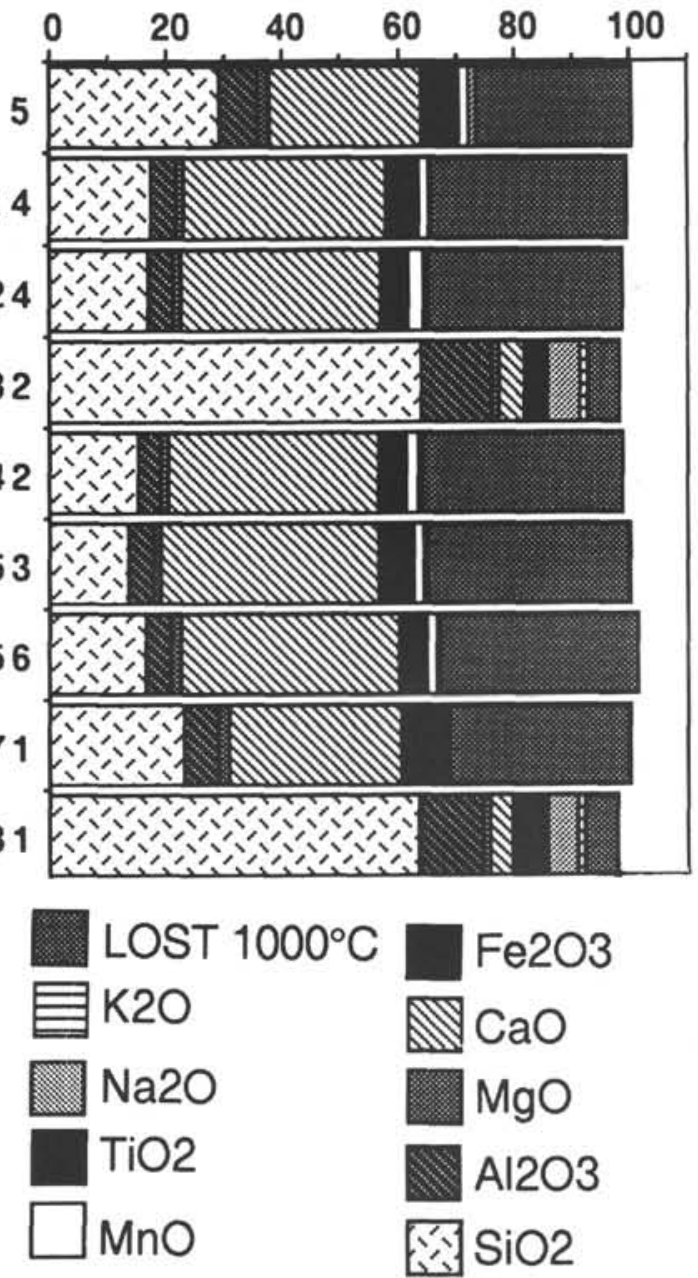

Figure 4. A. Cumulative histograms vs. depth of semiquantitative estimates of the solid phase abundances (\%) based on XRD diagrams, Hole 836A. B. Relative amounts of major chemical components in the bulk sediments (wt\%), Holes 836A and 837A. Parameters as in Figure 2.

suggesting a lower alteration grade, because the sediment is characterized by a higher content of reactive material, such as vitric glass. However, as discussed below, evidence is present of $\mathrm{Fe}-\mathrm{Mg}$ and transition element-bearing clays in these sites.

Effect of the Hydrothermal Activity on the Sediments: Evidence from Minor Elements

Phosphorous is an element that is easily mobilized in hydrothermal processes, as apatite, the most common phosphate-bearing min- eral, belongs to the accessory minerals of hydrothermal deposits. A useful indicator for determining the origin of apatite is yttrium, as this element is only fixed in organogenic apatite and is almost lacking in hydrothermal apatite (Marchig et al., 1982). In Figure 13, $\mathrm{P}_{2} \mathrm{O}_{5}$ is plotted against $\mathrm{Y}$ for all collected sediment samples from the Lau Basin and for sediments from Fiji Basin (Marchig et al., 1990). The domain for the sediments from Fiji Basin yields a shift of about $+0.2 \%$ $\mathrm{P}_{2} \mathrm{O}_{5}$ with respect to the biogenic apatite regression line. Marchig et al. (1990) think that this shift corresponds to the hydrothermal phosphorus taken up by the sediment. The Lau sediments present a dif- 
A

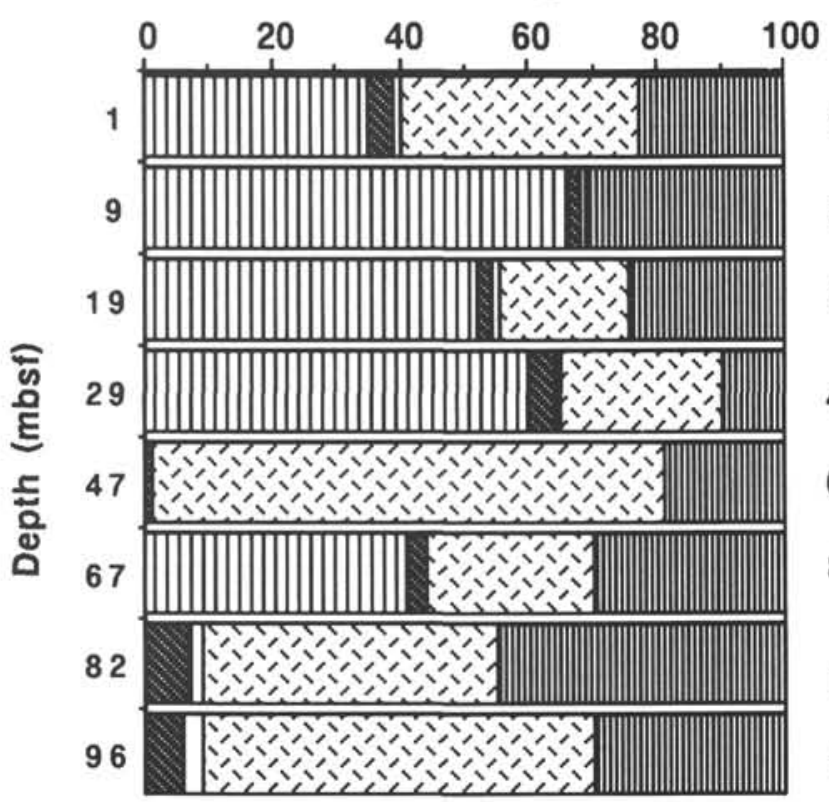

$1 \mathrm{H} 1$ (2)

$2 \mathrm{H} 4(1)$

$3 \mathrm{H} 4$ (2)

$4 \mathrm{H} 4$ (2)

$6 \mathrm{H} 4$ (3)

$8 \mathrm{H} 4$ (2)

$10 \mathrm{H} 2(3)$

$11 \mathrm{H} 5$ (3)

B

Site $838 \mathrm{~A}$ weight (\%)
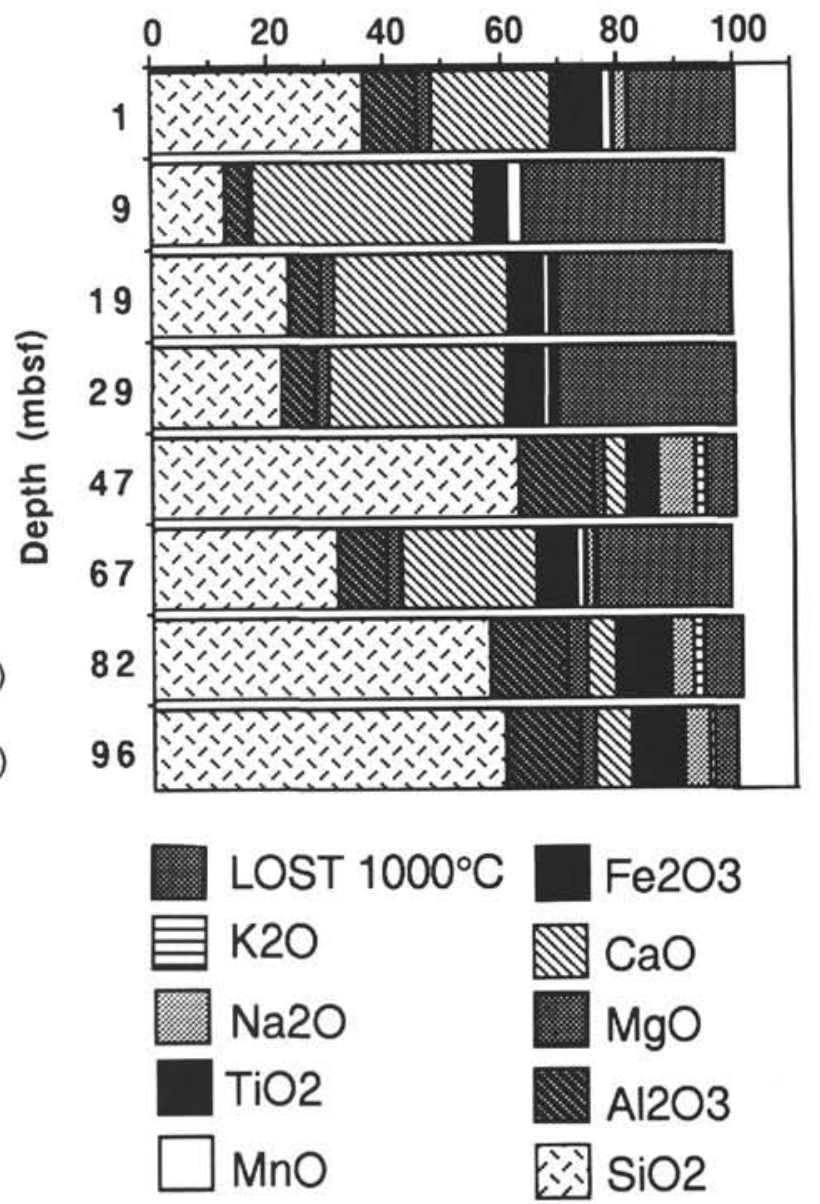

Figure 5. A. Cumulative histograms vs. depth of semiquantitative estimates of the solid phase abundances (\%) based on XRD diagrams, Hole 838 A. B. Relative amounts of major chemical components in the bulk sediments (wt\%), Hole 838A. Parameters as in Figure 2.

ferent distribution from that of the Fiji sediments. On a carbonate-free basis, sediments from Class 3 have the lowest contents in $\mathrm{P}_{2} \mathrm{O}_{5}$ and $\mathrm{Y}$, and lie between the biogenic apatite line and the domain of the Fiji sediments. Samples from Class 1 are the most enriched in $\mathrm{P}_{2} \mathrm{O}_{5}$ and $\mathrm{Y}$, and the majority of the samples lie on the line of the hydrothermal sediments. The $\mathrm{P}_{2} \mathrm{O}_{5}$ enrichment with respect to $\mathrm{Y}$ is at a maximum in samples mainly collected in Holes 834A and 835A. In agreement with previous studies, we assume that in these samples hydrothermal apatite exceeds apatite of biogenic origin.

$\mathrm{Cr}$ is partly mobile in hydrothermal processes as well as being enriched in hydrothermal precipitates, whereas other terrigenous elements ( $\mathrm{Ti}, \mathrm{K}, \mathrm{Mg}, \mathrm{Rb}$, and $\mathrm{Zr}$ ) are not. The plot of Ti vs. $\mathrm{Cr}$ (Fig. 14) does not show a statistically significant correlation $(r=0.165)$, which suggests that $\mathrm{Cr}$ in the Lau sediments is mainly of hydrothermal origin. This is in good agreement with the highest $\mathrm{Cr}$ concentrations, which reach a value of $50 \mathrm{mg} / \mathrm{kg}$ in the samples from Class 1 taken in Hole 834A (Table 2), and with the lowest average of $\mathrm{Cr}$ concentrations determined in the samples from Class 3 (i.e., $11.4 \pm 3.8 \mathrm{mg} / \mathrm{kg}$ ). However, the three deeper samples from Site 839, previously identified by the excess of $\mathrm{Al}$ with respect to $\mathrm{Si}$ (Fig. 9) and by $\mathrm{Mg}$ with respect to $\mathrm{Ca}$ (Fig. 9), were not considered in calculating this average. These samples also have the highest $\mathrm{Cr}$ concentrations and relatively high contents in transitional elements, such as $\mathrm{Zn}, \mathrm{Cu}, \mathrm{Ni}$, and $\mathrm{Co}$. Clay minerals probably act as a secondary sink for these elements. In each hole, Sc correlates highly with $\mathrm{Al}$ (Fig. 15), which suggests that Sc most probably is connected to the clay fraction of the sediments.
This has been previously observed in the Lau sediments from 0 to 700 k.y. (Kunzendorf et al., 1990).

The venting hydrothermal solutions at $21^{\circ} \mathrm{N}$ on the East Pacific Rise (EPR) and on the Valu Fa Ridge are rich in the ore-forming elements (OFE) Fe, $\mathrm{Mn}, \mathrm{Zn}, \mathrm{Cu}, \mathrm{Ni}$, and Co (Edmond et al., 1982; Von Damm et al., 1985; Fouquet et al., 1990, 1991). Hydrothermal and metalliferous Mn crusts of different ages from FAMOUS, Galapagos, Cyprus, Leg 54, the Bauer Deep, Marquesas Zone, and the North Pacific have been characterized by a comparison between the sum of $\mathrm{Cu}+\mathrm{Ni}+\mathrm{Co}$ and $\mathrm{REE}$ as well as by the $\mathrm{Nd}$ and $\mathrm{Sr}$ isotopic compositions (Clauer et al., 1984). For these reasons, the relationship between the sum of the ore-forming elements $(\mathrm{OFE}=\mathrm{Zn}+\mathrm{Cu}+\mathrm{Ni}$ $+\mathrm{Co}+\mathrm{Cr}$ ) and $\mathrm{Mn}$ is displayed in Figure $16 . \mathrm{Cr}$ is associated with the OFE because it is predominantly of hydrothermal origin. The sum of OFE correlates positively with $\mathrm{Mn}(\mathrm{OFE}=10.25 \mathrm{Mn}+158.8, r=$ 0.843 ), whereas $\mathrm{Zn}, \mathrm{Cu}, \mathrm{Ni}, \mathrm{Co}$, and $\mathrm{Cr}$ are not correlated individually with $\mathrm{Mn}(r<0.45)$. This suggests a common origin for the OFE and $\mathrm{Mn}$, whereas these elements do not behave similarly in seawater and sediments. The majority of the samples have OFE values that range from 100 to $350 \mathrm{mg} / \mathrm{kg}$ and $\mathrm{Mn}$ values that range from 1 to $15 \mathrm{~g} / \mathrm{kg}$. These values are comparable to the major deposits of hydrothermal sediments from the East Pacific Rise (Heath and Dymond, 1977; Lyle et al., 1986; Marchig and Erzinger, 1986; Schrader et al., 1980). Samples from Sites 834 and 835 , which have been previously identified in Figure 12, show higher OFE and Mn values that range from 350 to $850 \mathrm{mg} / \mathrm{kg}$ and from 15 to $60 \mathrm{~g} / \mathrm{kg}$, respectively. Comparable ranges 


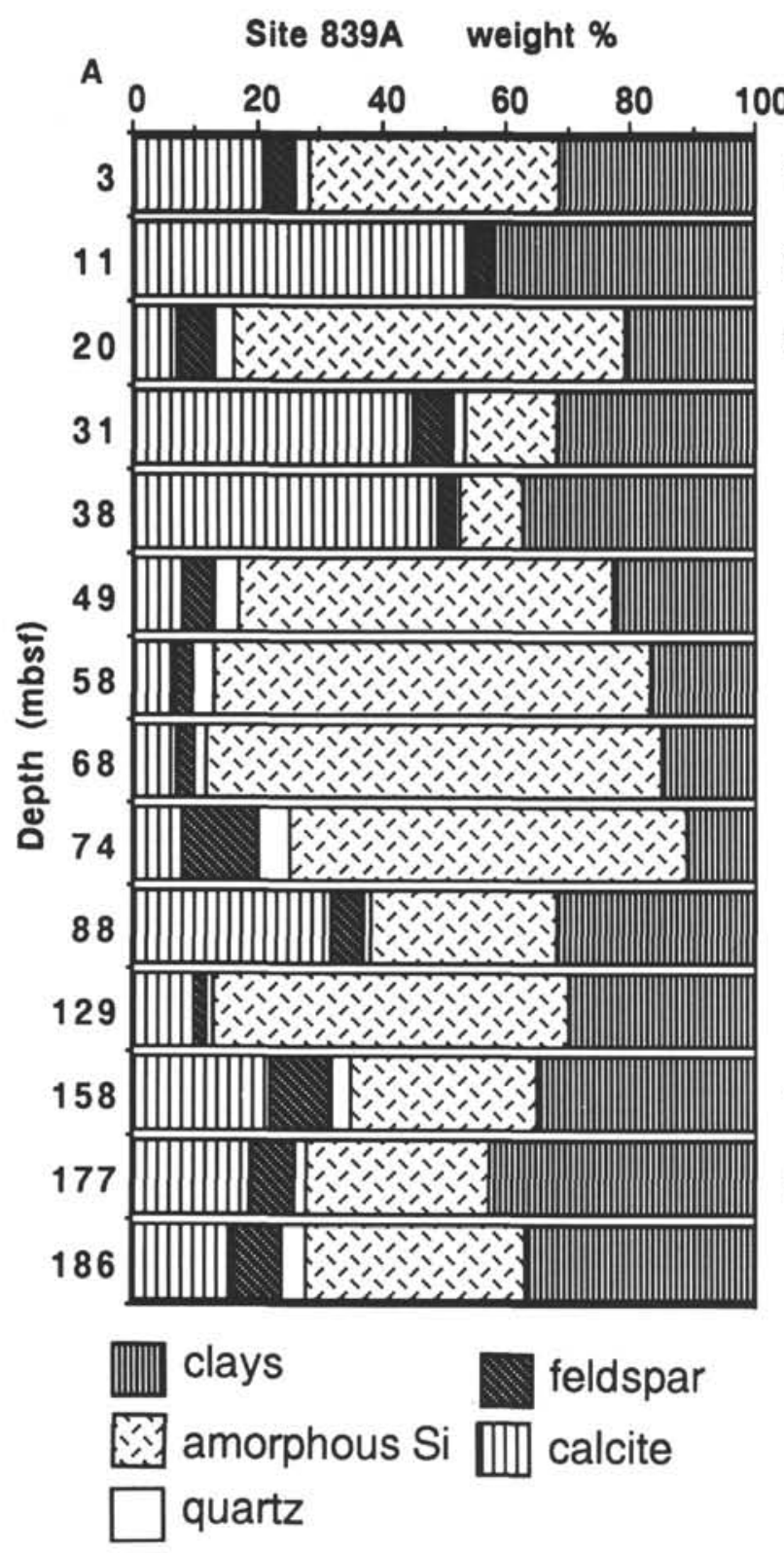

$1 \mathrm{H1}$ (3)

$2 \mathrm{H} 4$ (1)

$3 \mathrm{H} 4$ (3)

$4 \mathrm{H} 5$ (2)

$5 \mathrm{H} 4$ (2)

$6 \mathrm{H} 4$ (3)

7H4 (3)

$8 \mathrm{H} 4$ (3)

$9 \mathrm{H} 4$ (3)

$10 \mathrm{H} 5$ (2)

$15 \times 1$ (3)

$18 \times c c(3)$

$20 \times c c(3)$

$21 \mathrm{Xcc}(3)$

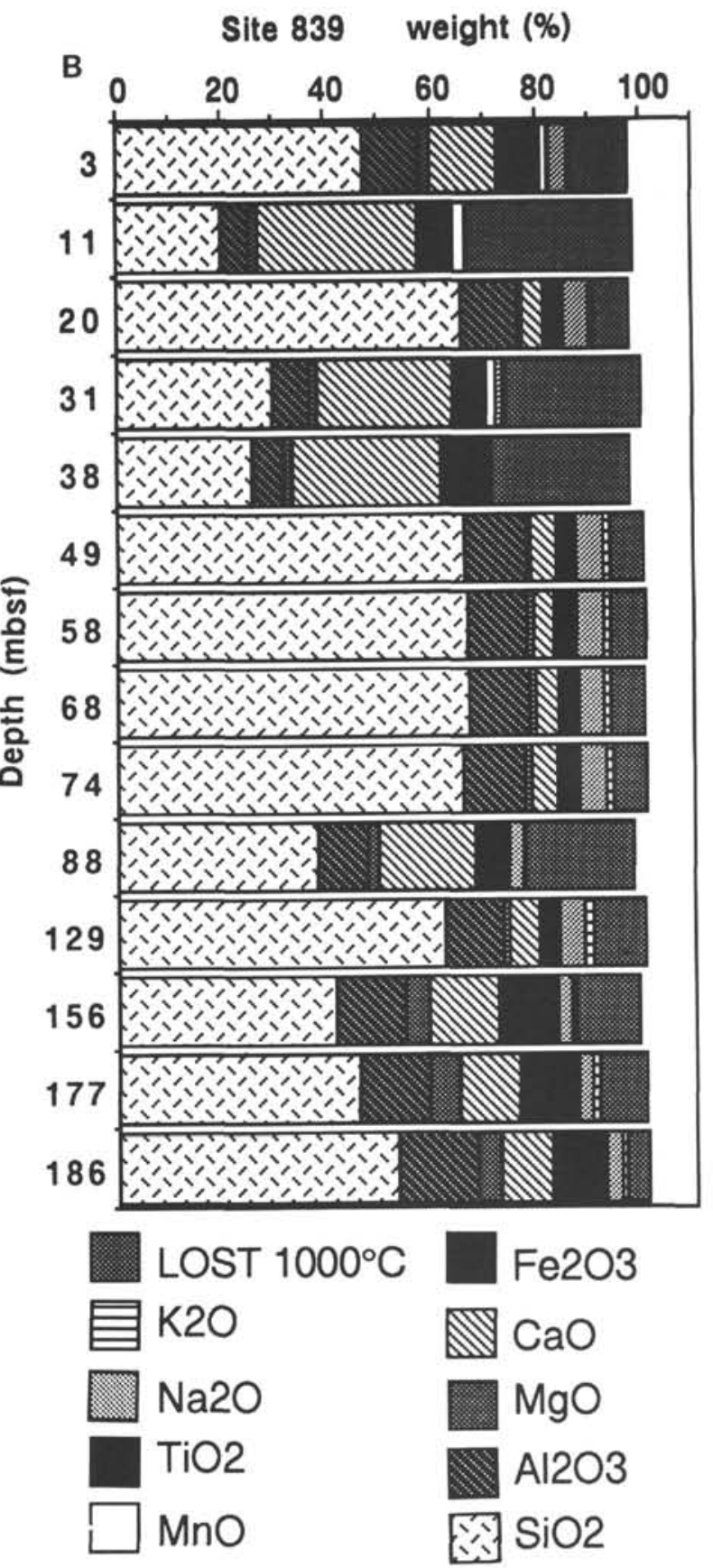

Figure 6. A. Cumulative histograms vs. depth of semiquantitative estimates of the solid phase abundances (\%) based on XRD diagrams, Hole 839A. B. Relative amounts of major chemical components in the bulk sediments (wt\%), Hole 839A. Parameters as in Figure 2.

of OFE and Mn concentrations have been reported in sediments from the Galapagos Ridge, the Bauer Deep, and at $9^{\circ} \mathrm{N}$ of EPR (Schrader et al., 1980, Heath, 1981). These high concentrations cannot be derived from extended contact between sediment and seawater, because OFEand Mn-enriched sediments accumulated at different rates (from 0.4 to $10 \mathrm{~cm} / \mathrm{k} . \mathrm{y}$.). Thus, to explain these high concentrations, inputs of exhalative components in the water column are required.

\section{Estimation of the Hydrothermal Fe/Mn Ratio}

A plot of the Fe/Mn ratios vs. the amounts of the OFE (Fig. 17) distinguishes four groups of samples having characteristic $\mathrm{Fe} / \mathrm{Mn}$ ratios of $45.9 \pm 2.6$ ( 4 samples), $25.3 \pm 2.8$ ( 6 samples), $12.8 \pm 2.0$ ( 8 samples), and $3.55 \pm 1.4$ (42 samples), including those with the highest OFE and Mn values. This latter average value for the Fe/Mn ratios is similar to that determined on the bulk sediments of the EPR (Dymond, 1981; Ruhlin and Owen, 1986; Marchig and Erzinger, 1986 ) and on the surficial sediments from 0 to 720 k.y. from the Lau Basin (Riech et al., 1990). With this in mind, we infer that the various OFE and Mn contents for this group of samples may reflect variations in the input of these components into the water column. This variation may be related to the intensity of hydrothermal activity in the northern $\mathrm{Lau}$ Basin. Furthermore, the $\mathrm{Fe} / \mathrm{Mn}$ ratios are relatively constant in samples collected at various depths (i.e., samples different in age), which suggests a relative uniformity in the composition of the hydrothermal sources. 
A

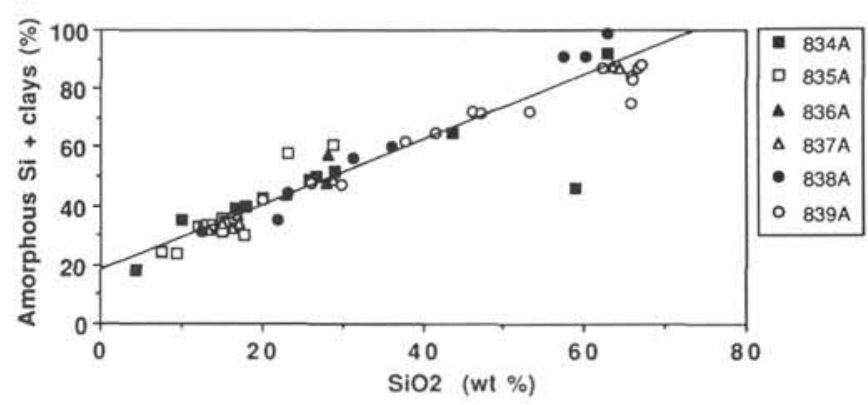

B

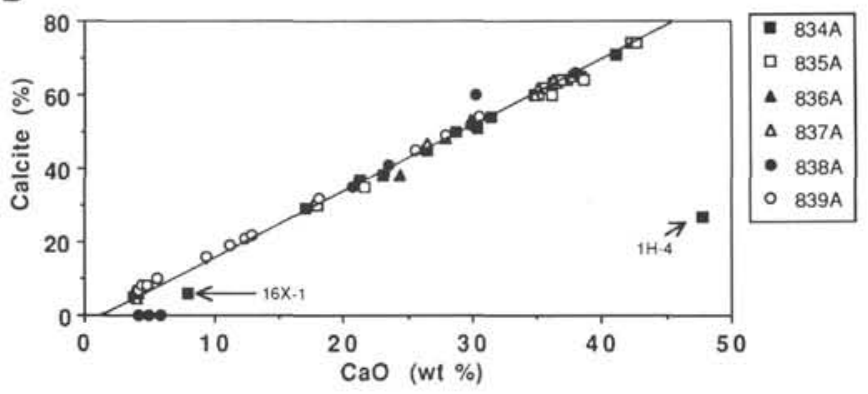

Figure 7. A. Amorphous silica + clays (\%) vs. $\mathrm{SiO}_{2}$ (wt\%). Sample 135-834A$16 \mathrm{X}-1,140-150 \mathrm{~cm}$, is excluded from the correlation line $(y=1.09 x+18.57$, $r=0.948$ ). B. Calcite (\%) vs. $\mathrm{CaO}(\mathrm{wt} \%)$. Sample 135-834A-1H-4, 140-150 $\mathrm{cm}$, is not included in the correlation line $(y=1.80 x-2.48, r=0.991)$.

The $\mathrm{Fe} / \mathrm{Mn}$ ratios average about 1.3 in the leachable oxides of the Lau Basin (Riech, 1990). The Fe/Mn ratio of the Class 1 samples is $2.8 \pm 0.8$ and is identical with that determined in the Quaternary carbonate-bearing sediments from the southern Lau Basin (Riech, 1990). Thus, the average of the $\mathrm{Fe} / \mathrm{Mn}$ ratios is probably close to 1.3 in the leachable oxides of the Class 1 samples. Higher Fe/Mn ratios are essentially given by the Class 3 samples, and cannot be derived directly from periodic inputs of volcanogenic materials, which alone cannot explain why the highest Fe concentrations in the Class 3 samples are not associated with the highest Si concentrations and amorphous silica contents (Figs. 2-6). In the Lau Basin, Mn occurs almost entirely in the easily reducible phase (Walter et al., 1990). Thus, considering that $\mathrm{Mn}$ in the bulk sediment has predominantly an exhalative origin, excess $\mathrm{Fe}$ in samples from Classes 2 and 3 can be estimated using the average value of the $\mathrm{Fe} / \mathrm{Mn}$ ratio of the Class 1 samples as follows:

$$
\mathrm{Fe}_{\text {excess }}=\mathrm{Fe}_{\text {total }}-\left[\mathrm{Mn}_{\text {sample }} \times(\mathrm{Fe} / \mathrm{Mn})_{\text {Class } 1}\right] .
$$

Values of excess Fe range from $20 \%$ to $95 \%$ of total $\mathrm{Fe}$. The highest values, ranging from $90 \%$ to $95 \%$, are determined in the Class 3 samples, and are related to the highest $\mathrm{Mg}$ and $\mathrm{Al}$ concentrations. Thus, diagenetic processes leading to the formation of $\mathrm{Fe}-\mathrm{Mg}$ bearing clays must be invoked to explain the enhanced $\mathrm{Fe}$ concentrations in the Class 3 samples and the periodic inputs of volcanogenic detritus are not the only source of higher values of $\mathrm{Fe} / \mathrm{Mn}$.

\section{Elementary Accumulation Rates: Comparison with Previous Data}

Elementary accumulation rates (EAR) are calculated in $\mathrm{mg} / \mathrm{cm}^{2} /$ k.y. using the following formula:

$$
\mathrm{EAR}=\mathrm{C} \times \mathrm{S} \times \rho \mathrm{T},
$$

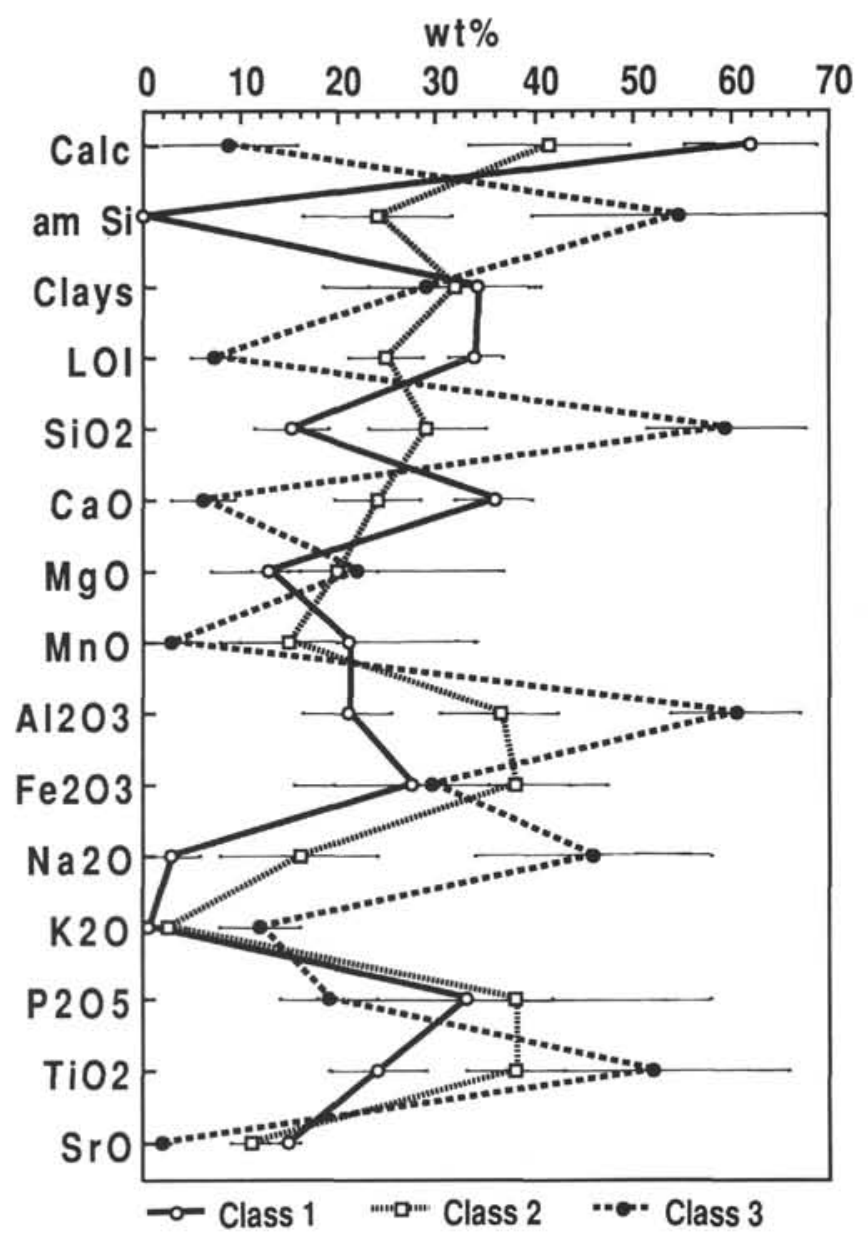

Figure 8. Contents of major chemical components and major solid phases for each class of samples. $\mathrm{Al}_{2} \mathrm{O}_{3}$ and $\mathrm{Fe}_{2} \mathrm{O}_{3}(\mathrm{wt} \% \times 5) ; \mathrm{MgO}, \mathrm{MnO}, \mathrm{Na}_{2} \mathrm{O}$, and $\mathrm{K}_{2} \mathrm{O}(\mathrm{wt} \% \times 10) ; \mathrm{P}_{2} \mathrm{O}_{5}, \mathrm{TiO}_{2}$, and $\mathrm{SrO}(\mathrm{wt} \% \times 100)$. Data from Table 3 .

where $\mathrm{C}=$ the element concentration of the sample $(\mathrm{mg} / \mathrm{g}), \mathrm{S}=$ the sedimentation rate $(\mathrm{cm} / \mathrm{k} \cdot \mathrm{y}$.$) , and \rho \mathrm{T}=$ the dry-bulk density $\left(\mathrm{g} / \mathrm{cm}^{3}\right)$. The values of the sedimentation rate and the dry-bulk density used here result from data obtained during Leg 135 (Parson, Hawkins, Allan, et al., 1992). The accumulation and sedimentation rates for each hole are presented in Table 4. Mn and OFE seem to be the best hydrothermal indicators, as Fe does not correlate with $\mathrm{Mn}(r=0.105)$ and is associated with various phases. $\mathrm{Mn}$ and OFE accumulation rates are widely scattered, resulting from variations in absolute $\mathrm{Mn}$ concentrations and sedimentation rates. In the $0-1.8 \mathrm{~m} . \mathrm{y}$. old sediments, the Mn accumulation rates range from 1 to $25 \mathrm{mg} / \mathrm{cm}^{2} / \mathrm{k} . \mathrm{y}$., and average about 12 . This value is similar to that about 13 , determined in Lau sediments collected during the SONNE-35 cruise (Walter et al., 1990). This Mn flux into the sediment is significantly higher than in the open ocean where only $0.2-2.1 \mathrm{mg} \mathrm{Mn} / \mathrm{cm}^{2} / \mathrm{k}$.y. accumulate (Elderfield, 1977). Sediments older than 1.8 m.y. (from 1.8 to 4 m.y.), and located closer to the hydrothermal activity, have considerably enhanced $\mathrm{Mn}$ accumulation rates that range from 25 to $198 \mathrm{mg} \mathrm{Mn} / \mathrm{cm}^{2} / \mathrm{k}$.y. Some of these values are higher than the highest value of about 40 , determined in sediments from $5^{\circ}$ to $20^{\circ} \mathrm{S}$ EPR (McMurtry et al., 1981), which suggests intensive hydrothermal activity during the spreading of the Lau Basin. Presently, the Valu Fa Ridge in the southern part of the Lau Basin is one of the most active areas in the world ocean (Fouquet et al., 1990, 1991). Mn and He anomalies were detected above the Valu Fa Ridge, and as far as $900 \mathrm{~m}$ above the seafloor (Von Stackelberg et al., 1988). Thus, the hydrothermal plume 
Table 3. Average values for the contents in major solid phase and for major element chemistry of three classes of bulk sediment.

\begin{tabular}{|c|c|c|c|c|c|c|}
\hline \multirow{4}{*}{$\begin{array}{c}\text { Calcite } \\
\text { Amorphous } \mathrm{Si}\end{array}$} & \multicolumn{2}{|c|}{ CLASS 1} & \multicolumn{2}{|c|}{ CLASS 2} & \multicolumn{2}{|c|}{ CLASS 3} \\
\hline & \multicolumn{2}{|c|}{$\begin{array}{l}\text { Biogenic calcite-rich } \\
\text { sediment }(\%) n=26\end{array}$} & \multicolumn{2}{|c|}{$\begin{array}{l}\text { Biogenic calcite-volcanoclastic } \\
\text { mixture }(\%) n=16\end{array}$} & \multicolumn{2}{|c|}{$\begin{array}{l}\text { Volcanogenic-rich } \\
\text { sediment }(\%) n=16\end{array}$} \\
\hline & $62.0+1-6.7$ & $(50-75)$ & $41.6+/-8.1$ & $(30-60)$ & $8.7+/-7.1$ & $(0-20)$ \\
\hline & 0.0 & & $24.0+1-7.6$ & $(10-30)$ & $54.8+/-15.0$ & $(30-80)$ \\
\hline Clays & $34.3+/-5.7$ & $(20-45)$ & $31.8+/-8.8$ & $(20-50)$ & $28.9+/-10.5$ & $(15-45)$ \\
\hline $\mathrm{SiO} 2$ & $15.2+/-3.8$ & $(07-27)$ & $29.1+/-5.9$ & $(21-44)$ & $59.6+/-8.1$ & $(42-67)$ \\
\hline $\mathrm{Al} 2 \mathrm{O} 3$ & $4.2+1-0.9$ & $(2.5-7.7)$ & $7.3+/-1.2$ & $(5.8-9.6)$ & $12.1+/-1.3$ & $(10.7-15.2)$ \\
\hline $\mathrm{MgO}$ & $1.3+/-0.2$ & $(0.9-1.9)$ & $2.0+1-0.4$ & $(1.3-2.7)$ & $2.2+/-1.5$ & $(1.1-5.5)$ \\
\hline $\mathrm{CaO}$ & $35.9+/-3.9$ & $(26-43)$ & $24.1+/-4.4$ & $(17-30)$ & $6.2+/-3.2$ & $(3-13)$ \\
\hline $\mathrm{Fe} 2 \mathrm{O} 3$ & $5.5+/-1.6$ & $(3.0-10.3)$ & $7.6+/-1.9$ & $(4.7-12.8)$ & $5.9+/-2.8$ & $(3.3-10.6)$ \\
\hline $\mathrm{P} 2 \mathrm{O} 5$ & $0.33+/-0.09$ & $(0.24-0.66)$ & $0.38+/-0.2$ & $(0.20-0.99)$ & $0.19+/-0.05$ & $(0.14-0.66)$ \\
\hline $\mathrm{MnO}$ & $2.3+/-1.4$ & $(1.02-5.75)$ & $2.0+/-1.9$ & $(0.37-8.48)$ & $0.3+1-0.3$ & $(0.14-1.37)$ \\
\hline TiO2 & $0.24+/-0.05$ & $(0.17-0.43)$ & $0.38+1-0.05$ & $(0.30-0.49)$ & $0.52+/-0.14$ & $(0.36-0.82)$ \\
\hline $\mathrm{SrO}$ & $0.15+/-0.01$ & $(0.13-0.17)$ & $0.11+1-0.02$ & $(0.08-0.15)$ & $0.02+1-0.01$ & $(0.01-0.05)$ \\
\hline $\mathrm{Na} 2 \mathrm{O}$ & $0.3+/-0.3$ & $(0.05-0.14)$ & $1.6+/-0.8$ & $(0.6-3.6)$ & $4.6+/-1.2$ & $(2.7-6.5)$ \\
\hline $\mathrm{K} 2 \mathrm{O}$ & $0.06+1-0.03$ & $(0.05-0.17)$ & $0.26+/-0.19$ & $(0.07-0.90)$ & $1.2+/-0.4$ & $(0.62-1.91)$ \\
\hline LOI & $34.0+1-2.8$ & $(25.3-39.4)$ & $24.9+/-3.7$ & $(18.6-31.6)$ & $7.2+/-2.3$ & $(4.0-12.0)$ \\
\hline
\end{tabular}

Notes: $\mathrm{LOI}=$ loss on ignition $\left(1000^{\circ} \mathrm{C}\right)$. Values in brackets correspond to the lowest and highest contents. See Figure 8.

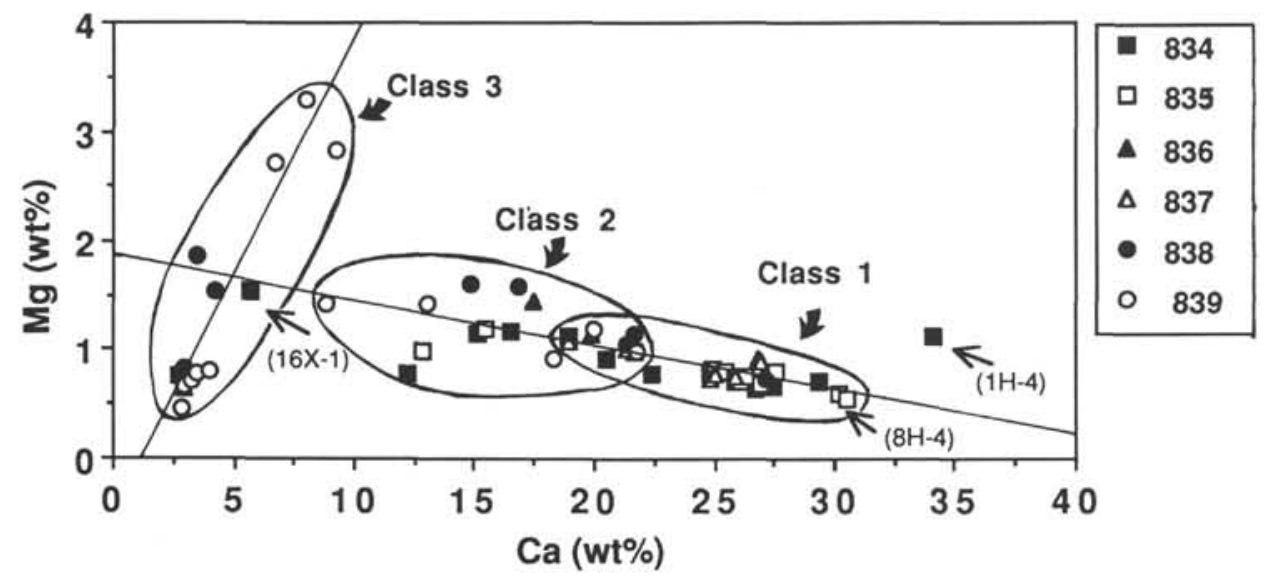

Figure 9. Mg vs. Ca for all bulk sediment samples analyzed.

rises to a considerable height, with a high $\mathrm{Mn}$ concentration generated in the water mass of the Lau Basin. The mineralizations observed at the Valu Fa Ridge in the Lau Basin appear to cover larger areas than those of the EPR (Von Stackelberg et al., 1990). Hydrothermal precipitates probably spread over the entire Lau Basin.

\section{Relationship between Chemical Accumulation Rates and Distance to the Spreading Centers: \\ Contrasts Between the Northern and Central Areas of the Lau Basin}

The $\mathrm{Mn}$ and $\mathrm{OFE}$ accumulation rates were compared to the distance between the Lau Spreading Center and the location of the sediments from both the northern (Sites 834-835) and central (Sites 838-839) areas of the Lau Basin (Figs. 18-19). In each hole, depth in the sediment was converted to distance from the ELSC and CLSC by using established age-depth scales (Parson, Hawkins, Allan, et al., 1992) and assuming a constant spreading rate (i.e., $3.5 \mathrm{~cm} / \mathrm{yr}$ ). The assumption of a constant spreading rate may introduce small uncertainties, but they are not especially important for this comparison. Figures 18 and 19 show that the increasing distance to the active ridge because of seafloor spreading is reflected in a generally decreasing accumulation of Mn and OFE in both the northern and central areas of the Lau Basin. Hydrothermal precipitates are also more concentrated near the ridge. Therefore, higher $\mathrm{Mn}$ and $\mathrm{OFE}$ accumulation rates on the western flank of the ELSC are mainly recorded between 0 and $5 \mathrm{~km}$ off the ridge axis, whereas those on the western flank of the CLSC are recorded as far as $30 \mathrm{~km}$ away from the ridge axis. Even at this distance, these rates remain 2 to 10 times greater than those of the ELSC at equivalent distances from the ridges. This distribution cannot be explained by differences in the sedimentation rates because they are similar in both areas. Previous studies have shown that diagenetic remobilization of $\mathrm{Mn}$ in the sediment column also can be ruled out (Riech, 1990). This observation must be related, therefore, to higher $\mathrm{Mn}$ and OFE concentrations in the water mass of the northern part than in that of the central part of the Lau Basin. This hypothesis can 


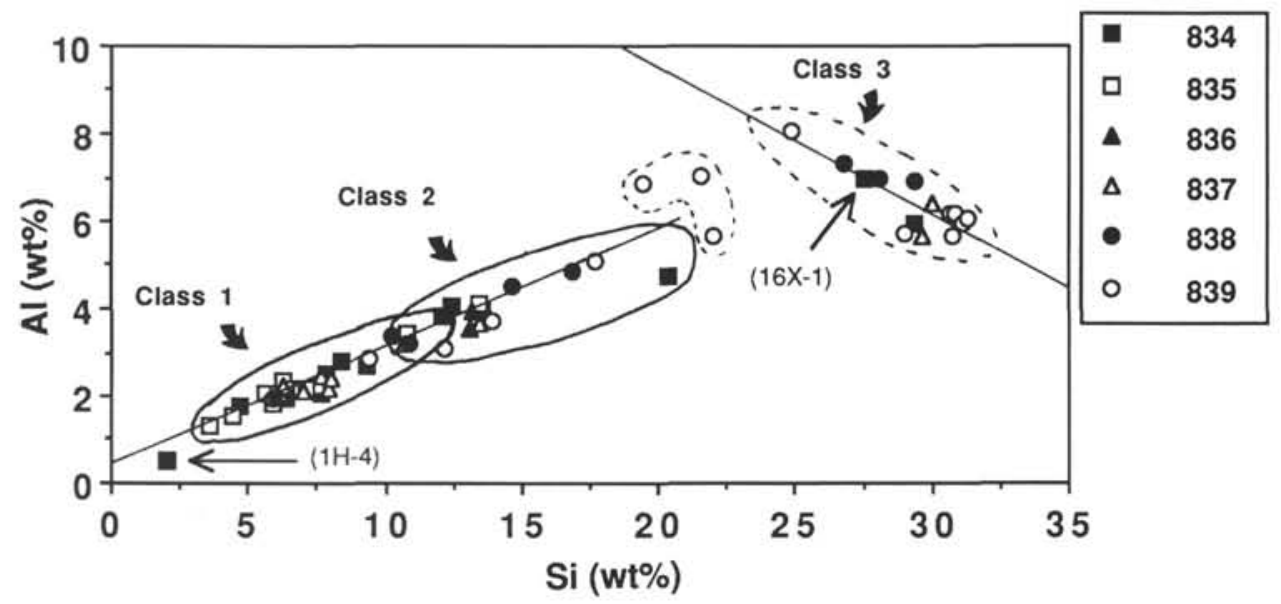

Figure 10. Al vs. Si for all bulk sediment samples analyzed.

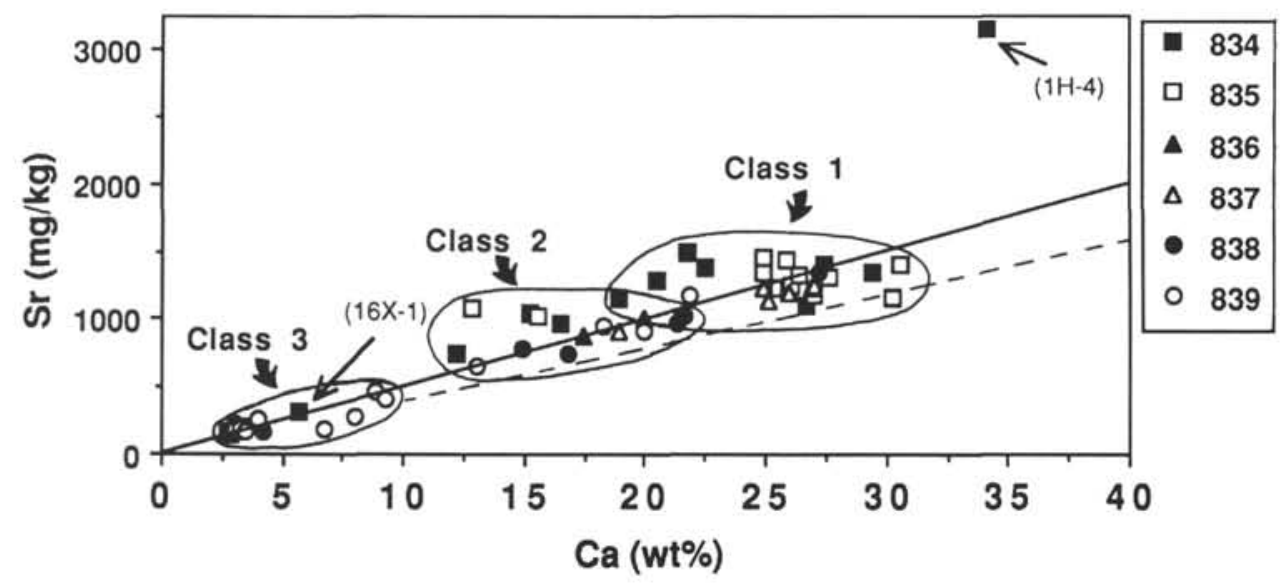

Figure 11. Sr vs. Ca for all bulk sediment samples analyzed. Dashed line $=\mathrm{Sr} / \mathrm{Ca}$ relationship in calcareous oozes between 0 and $4 \mathrm{Ma}$ (after Baker et al., 1982).

be tested by considering the accumulation rates of all sediment fractions recorded over $10 \mathrm{~km}$ for Holes $834 \mathrm{~A}$ and $835 \mathrm{~A}$, and over 5 $\mathrm{km}$ for Holes $838 \mathrm{~A}$ and $839 \mathrm{~A}$.

\section{Quantitative Assessment of the Hydrothermal Fraction: Hydrothermal Ponded Sediments}

The average values of the accumulation rates of the carbonate fraction (CF), the hydrothermal fraction (HF), and the remaining fraction have been calculated for Holes $834 \mathrm{~A}$ and $835 \mathrm{~A}$, and for Holes $838 \mathrm{~A}$ and $839 \mathrm{~A}$. The accumulation rate of the $\mathrm{HF}$ has been estimated using the $\mathrm{MnO}$ and the $\mathrm{Fe}_{2} \mathrm{O}_{3}$ accumulation rates deduced from the $\mathrm{Fe}_{2} \mathrm{O}_{3} / \mathrm{MnO}$ ratios of the leachable oxides, which average about 1.4 (Riech, 1990). The OFE accumulation rate can be discarded for this calculation. The accumulation rate of the remaining fraction, corresponding to that for the volcanic detritus and authigenic minerals (VAMF), is estimated by the difference between the noncarbonate fraction and hydrothermal fraction. The results shown in Table 5 indicate that the accumulation rates of $\mathrm{HF}$ and $\mathrm{CF}$ at Sites 834 and 835 are 3-6 times and 2-4 times higher than those at Sites 838 and 839, respectively. This means that related processes favored the HF and $\mathrm{CF}$ accumulation. The contrast of the $\mathrm{HF}$ accumulation rates between the central and the northern part of the basin might be explained by greater previous hydrothermal activity within the CLSC. This hy- pothesis cannot be tested because no information is available on the past and present hydrothermal activities within these two spreading centers. However, the present hydrothermal activity recognized within the Valu Fa Spreading Center (VFSC) does not increase the Mn accumulation rates drastically in the recent sediment from the southern (i.e., $3 \mathrm{mg} / \mathrm{cm}^{2} / \mathrm{k}$.y.; Walter et al., 1990) and central parts (i.e., $5 \mathrm{mg} /$ $\mathrm{cm}^{2} / \mathrm{k}$.y. at $0.5 \mathrm{mbsf}$ at Site 838 ) of the Lau Basin. Furthermore, the $\mathrm{Fe} / \mathrm{Mn}$ ratios determined in this study suggest a relative uniformity of the hydrothermal sources. Contrasts in the $\mathrm{CF}$ accumulation rates cannot be directly derived from contrasts in the hydrothermal activity through time. Hence, an additional process must be involved to explain the higher $\mathrm{HF}$ and $\mathrm{CF}$ accumulation rates in the sediment from the northern part of the Lau Basin. This can be best understood in terms of ponding sediments in the basins where Holes 834A and 835A were drilled, and erosion of thinner and lighter particles by bottom currents during the sedimentation of the central area in the Lau Basin. Sedimentation rates in Holes $838 \mathrm{~A}$ and $839 \mathrm{~A}$ were compensated by increased inputs of coarser grained volcanic detritus, which were less easily reworked over a long distance than the carbonate, hydrothermal, and other thin particles. This assumption is substantiated by various independent observations. The lithofacies at Sites 838 and 839 are generally coarser grained than at Sites 834 and 835 . Furthermore, the maximum grain sizes of discrete ash layers and ash turbidites from the southern sites are almost systematically higher than those of their 


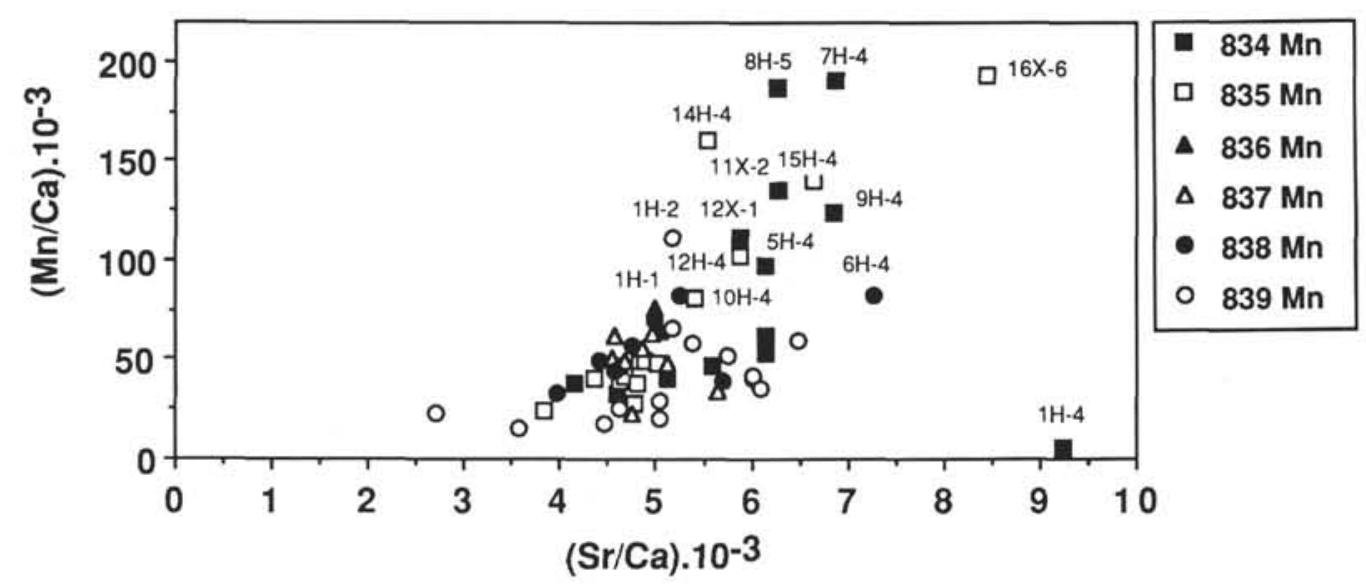

Figure 12. Mn/Ca vs. $\mathrm{Sr} / \mathrm{Ca}$ variation diagram for all bulk sediment samples analyzed.

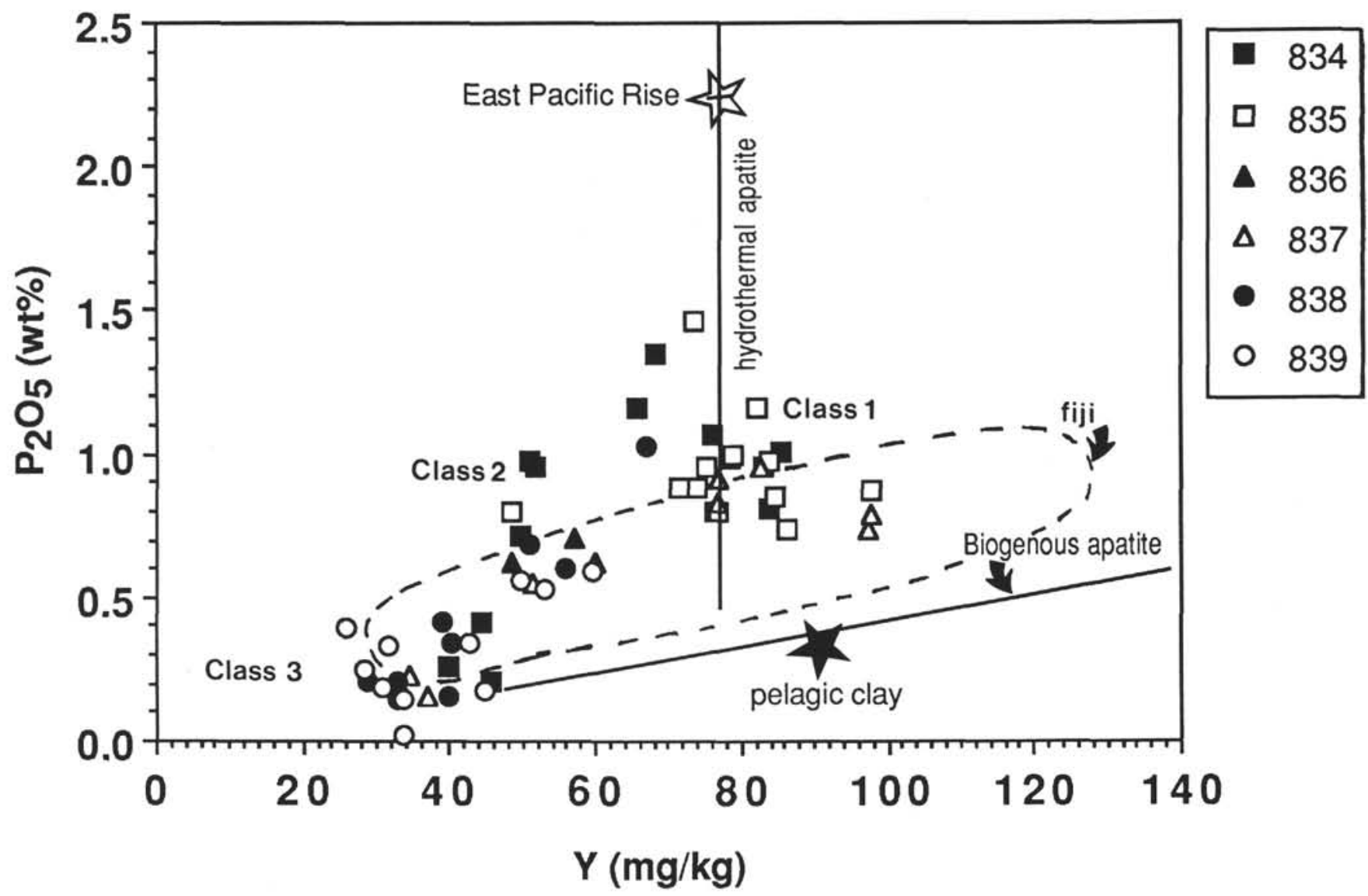

Figure 13. $\mathrm{P}_{2} \mathrm{O}_{5}$ vs. Y diagram for all sediments analyzed from the Lau Basin (all on carbonate-free basins). Elongated domain defines sediments from the Fiji basin (Marchig et al., 1990). The plotted regression lines show the relationship between $\mathrm{P}_{2} \mathrm{O}_{5}$ and $\mathrm{Y}$ in hydrothermal sediment and in apatite of organogenic origin (from Marchig et al., 1990). Open star = mean value of hydrothermal sediments from EPR, and solid star = mean value for deep-sea clay.

counterparts from the northern sites (Parson, Hawkins, Allan, et al., 1992). The average values for grain size are $268 \pm 112 \mu \mathrm{m}$ at Site 834 and $719 \pm 343 \mu \mathrm{m}$ at Site 839 . The selective erosion has chemical consequences. As zircons are heavy minerals, $\mathrm{Zr}$ concentrations in the sediment seem to be controlled by grain size (Fig. 20). A ${ }^{230} \mathrm{Th}$ depth profile study suggests that the proportion of ponding or focusing sediments could be at least $50 \%$ of the total accumulation in a core from the southern Lau Basin (Mangini and Stoffers, 1990). All clay and pelagic nannofossil oozes recovered at the backarc sites are stained a distinctive reddish brown by hydrothermally derived oxyhydroxides. Locally, sedimentation rates can be lower than $1 \mathrm{~cm} / \mathrm{k} . \mathrm{y}$. at Sites 839 and 835 (Parson, Hawkins, Allan, et al., 1992). All these independent data support the hypothesis of ponding sediments in the Lau Basin. Selective erosion followed by redeposition of ponding sediments could explain the more advanced diagenesis in the Holes $834 \mathrm{~A}$ and $835 \mathrm{~A}$ (northern area of the Lau Basin) than that observed 


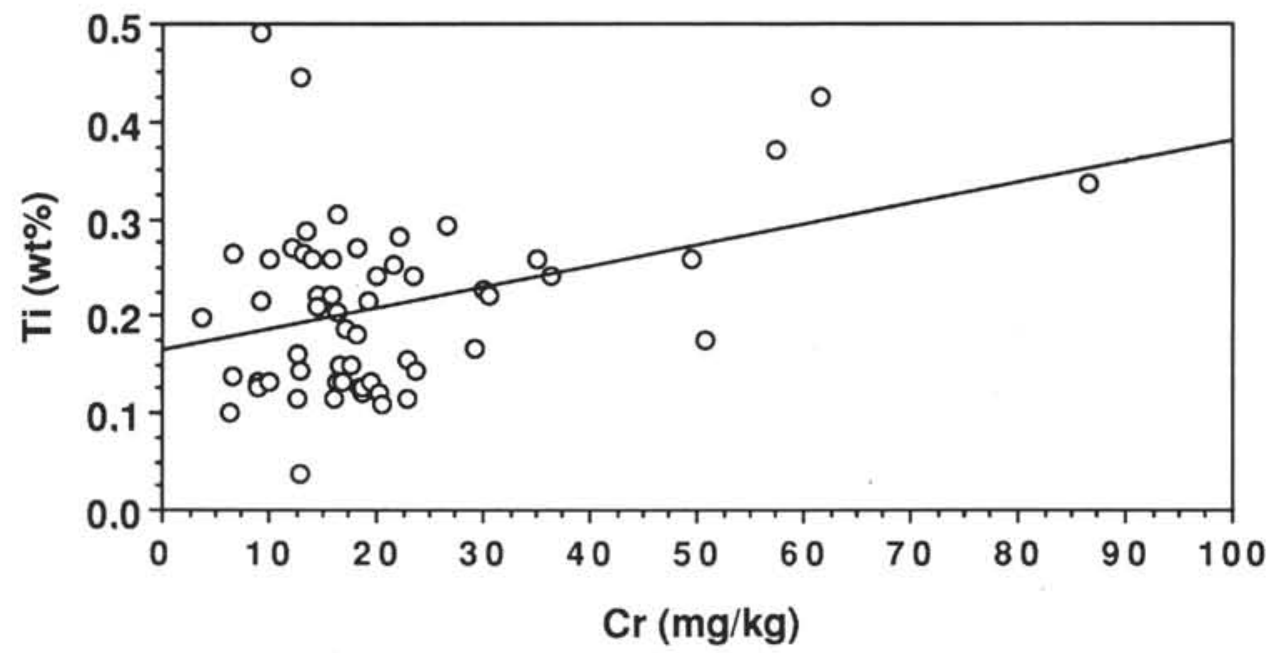

Figure 14. Ti vs. Cr diagram for all bulk sediment samples.

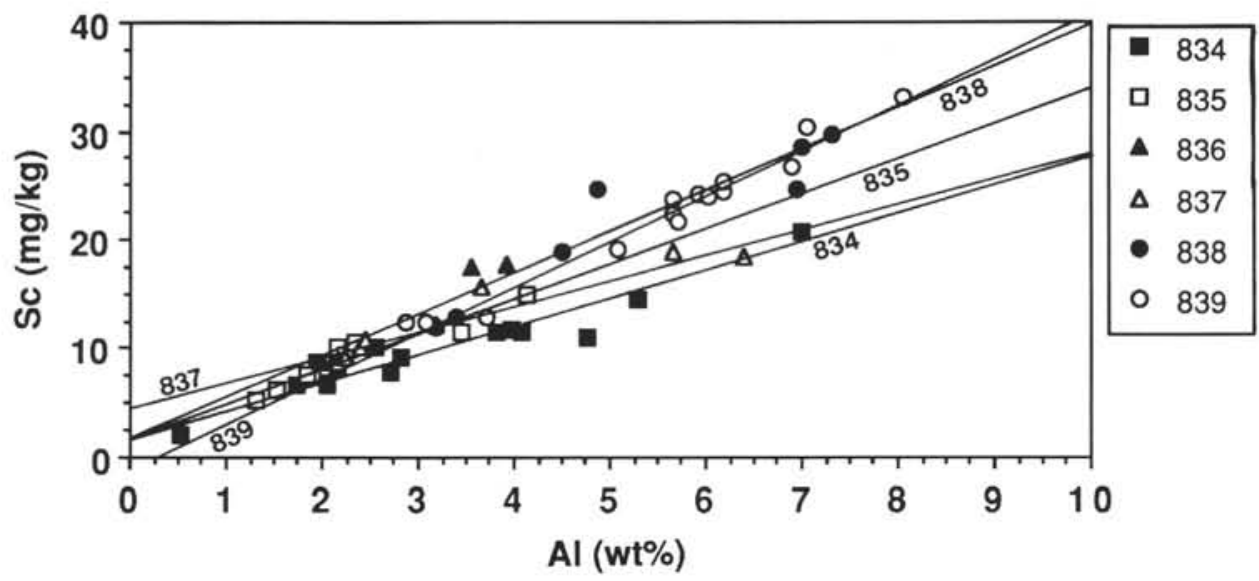

Figure 15. Sc vs. Al diagram for all bulk sediment samples. At each hole, the highest correlation coefficient has been determined by means of the following regression lines: Hole 834A, $\mathrm{Sc}=2.45 \mathrm{Al}+2.04, r=0.915$; Hole 835A, $\mathrm{Sc}=3.10 \mathrm{Al}+2.05, r=0.902 ;$ Hole $837 \mathrm{~A}, \mathrm{Sc}=2.41 \mathrm{Al}+4.42, r=0.921 ;$ Hole 838A, $\mathrm{Sc}=3.81 \mathrm{Al}+1.29, r=$ 0.917; and Hole 839A, $\mathrm{Sc}=4.20 \mathrm{Al}+1.13, r=0.912$.

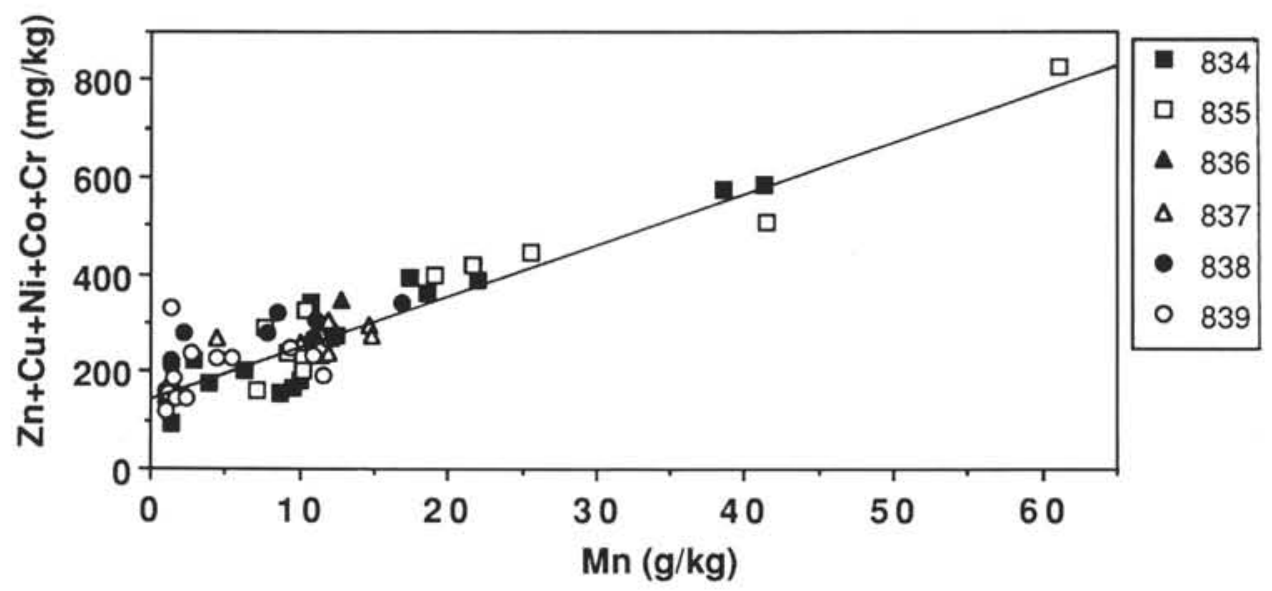

Figure 16. Ore-forming elements $(\mathrm{OFE})=\mathrm{Zn}+\mathrm{Cu}+\mathrm{Ni}+\mathrm{Co}+\mathrm{Cr}$ vs. Mn for all bulk sediment samples analyzed. 


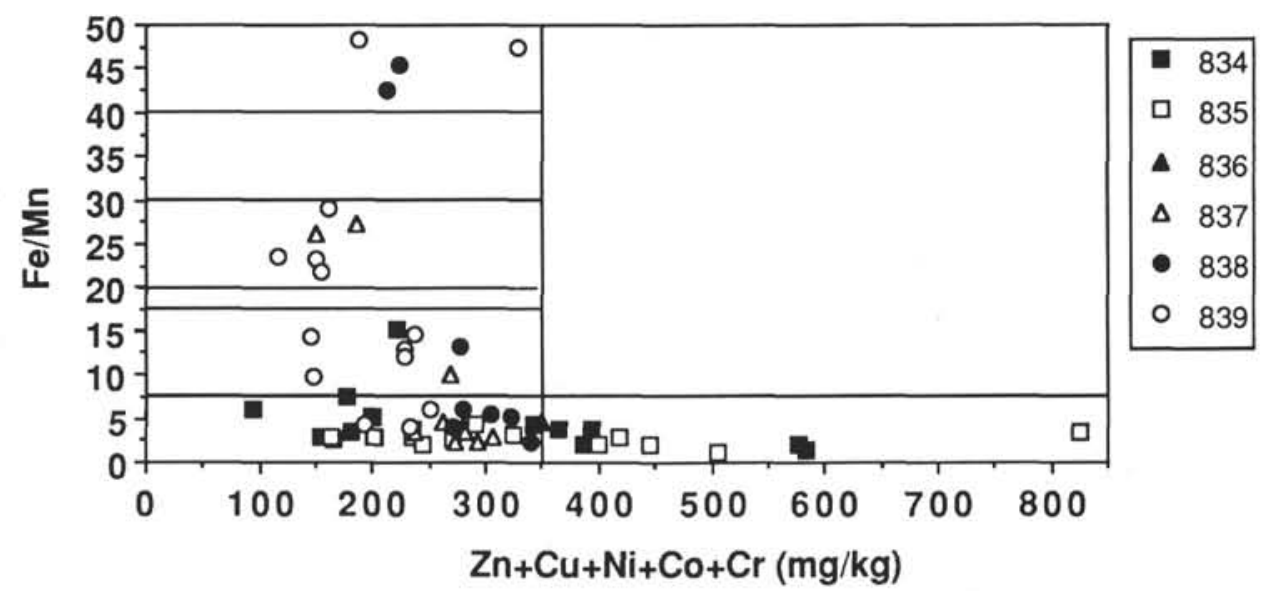

Figure 17. Variation diagram of $\mathrm{Fe} / \mathrm{Mn}$ vs. ore-forming elements $(\mathrm{OFE})=\mathrm{Zn}+\mathrm{Cu}+\mathrm{Ni}+\mathrm{Co}+\mathrm{Cr}$ for all bulk sediment samples analyzed.
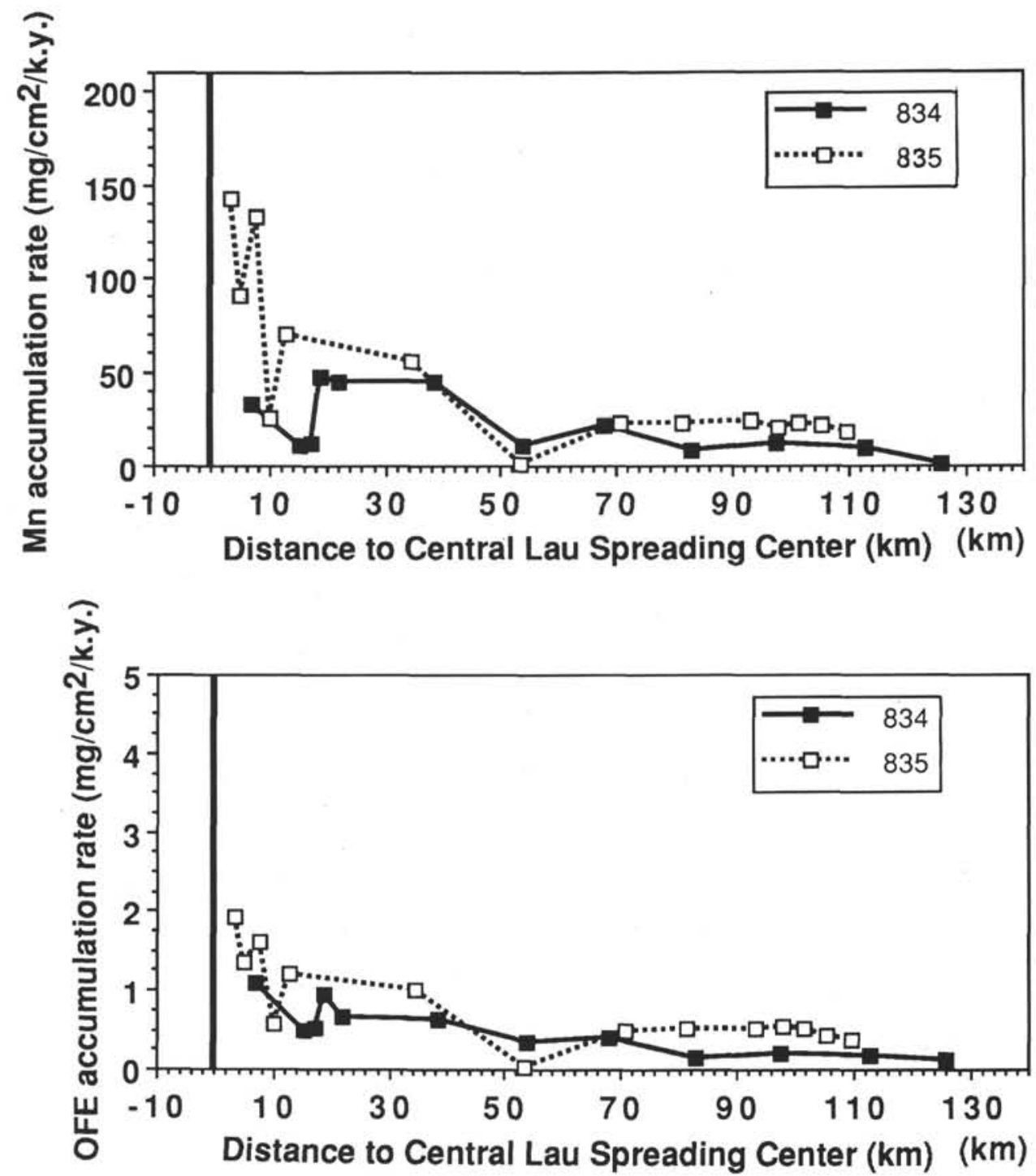

Figure 18. Manganese and ore-forming element accumulation rates vs. distance to the Central Lau Spreading Center at Sites 834 and 835 (northern area of the Lau Basin). 
Table 4. Accumulation rates of manganese and ore-forming elements (OFE) and sedimentation rates in Holes $834 \mathrm{~A}, 835 \mathrm{~A}, 837 \mathrm{~A}, 838 \mathrm{~A}$, and $839 \mathrm{~A}$.

\begin{tabular}{|c|c|c|c|c|}
\hline \multirow[b]{2}{*}{$\begin{array}{l}\text { Core-section } \\
\text { Interval }(\mathrm{cm})\end{array}$} & \multirow[b]{2}{*}{$\begin{array}{l}\text { Depth } \\
\text { (mbsf) }\end{array}$} & \multirow{2}{*}{\begin{tabular}{|c|}
$\begin{array}{c}\text { Sedimentation } \\
\text { rate } \\
(\mathrm{cm} / \mathrm{ky})\end{array}$ \\
\end{tabular}} & \multicolumn{2}{|c|}{ Accumulation rate } \\
\hline & & & $\mathrm{Mn}$ & $\left.\right|_{(\mathrm{mg} / \mathrm{cm} 2 / \mathrm{ky})} ^{\text {OFE }}$ \\
\hline 135-834A-1H-4, 140-150 & 5.9 & 3.2 & 2 & 0.10 \\
\hline $135-834 \mathrm{~A}-2 \mathrm{H}-4,140-150$ & 13.5 & 3.2 & 10 & 0.17 \\
\hline $135-834 \mathrm{~A}-3 \mathrm{H}-4,140-150$ & 23.0 & 3.2 & 12 & 0.21 \\
\hline $135-834 \mathrm{~A}-4 \mathrm{H}-4,140-150$ & 32.5 & 3.2 & 8 & 0.15 \\
\hline $135-834 \mathrm{~A}-5 \mathrm{H}-4,140-150$ & 42.0 & 3.2 & 22 & 0.40 \\
\hline $135-834 \mathrm{~A}-6 \mathrm{H}-4,140-150$ & 51.0 & 3.2 & 11 & 0.34 \\
\hline $135-834 \mathrm{~A}-7 \mathrm{H}-4,130-132$ & 60.9 & 3.2 & 45 & 0.64 \\
\hline $135-834 \mathrm{~A}-8 \mathrm{H}-5,78-80$ & 71.4 & 3.2 & 44 & 0.66 \\
\hline $135-834 \mathrm{~A}-9 \mathrm{H}-4,140-150$ & 80.0 & 6.4 & 47 & 0.93 \\
\hline $135-834 A-10 X-2,89-91$ & 86.0 & 6.4 & 12 & 0.51 \\
\hline $135-834 A-11 X-2,71-73$ & 95.4 & 6.4 & 11 & 0.49 \\
\hline $135-834 A-12 X-1,140-150$ & 104.3 & 6.4 & 32 & 1.08 \\
\hline $135-835 \mathrm{~A}-1 \mathrm{H}-4,140-150$ & 6.0 & 6.3 & 17 & 0.38 \\
\hline $135-835 \mathrm{~A}-2 \mathrm{H}-4,140-150$ & 15.5 & 6.3 & 21 & 0.42 \\
\hline $135-835 \mathrm{~A}-3 \mathrm{H}-4,140-150$ & 25.0 & 6.3 & 23 & 0.51 \\
\hline $135-835 \mathrm{~A}-4 \mathrm{H}-4,140-150$ & 34.0 & 6.3 & 21 & 0.53 \\
\hline $135-835 \mathrm{~A}-5 \mathrm{H}-5,90-92$ & 44.9 & 6.3 & 24 & 0.53 \\
\hline $135-835 \mathrm{~A}-6 \mathrm{H}-4,140-150$ & 53.5 & 6.3 & 23 & 0.51 \\
\hline $135-835 \mathrm{~A}-8 \mathrm{H}-4,140-150$ & 72.5 & 6.3 & 22 & 0.48 \\
\hline 135-835A-9H-3, 70-72 & 76.7 & 0.4 & 1 & 0.04 \\
\hline $135-835 \mathrm{~A}-10 \mathrm{H}-4,140-150$ & 91.5 & 9.7 & 55 & 1.02 \\
\hline $135-835 \mathrm{~A}-12 \mathrm{H}-4,140-150$ & 110.5 & 9.7 & 70 & 1.21 \\
\hline $135-835 \mathrm{~A}-13 \mathrm{H}-4,110-112$ & 119.6 & 9.7 & 25 & 0.57 \\
\hline $135-835 \mathrm{~A}-14 \mathrm{H}-4,140-150$ & 129.5 & 9.7 & 133 & 1.62 \\
\hline $135-835 \mathrm{~A}-15 \mathrm{H}-4,121-123$ & 138.7 & 9.7 & 91 & 1.34 \\
\hline 135-835A-16X-6, 140-150 & 148.5 & 9.7 & 142 & 1.92 \\
\hline $135-837 \mathrm{~A}-1 \mathrm{H}-3,140-150$ & 4.5 & 3.8 & 10 & 0.25 \\
\hline $135-837 \mathrm{~A}-2 \mathrm{H}-4,140-150$ & 14.0 & 3.8 & 12 & 0.23 \\
\hline $135-837 \mathrm{~A}-3 \mathrm{H}-4,140-150$ & 23.5 & 3.8 & 15 & 0.30 \\
\hline $135-837 \mathrm{~A}-4 \mathrm{H}-4,140-150$ & 31.5 & 3.8 & 2 & 0.24 \\
\hline $135-837 \mathrm{~A}-5 \mathrm{H}-4,130-132$ & 42.3 & 3.8 & 22 & 0.41 \\
\hline $135-837 \mathrm{~A}-6 \mathrm{H}-4,140-150$ & 53.5 & 3.8 & 14 & 0.33 \\
\hline 135-837A-7H-1, 18-20 & 55.7 & 3.8 & 13 & 0.34 \\
\hline $135-837 \mathrm{~A}-8 \mathrm{H}-4,140-150$ & 71.0 & 3.8 & 5 & 0.30 \\
\hline $135-837 \mathrm{~A}-9 \mathrm{H}-5,78-80$ & 81.3 & 3.8 & 1 & 0.19 \\
\hline $135-838 \mathrm{~A}-1 \mathrm{H}-1,50-52$ & 0.51 & 2.1 & 5 & 0.13 \\
\hline $135-838 \mathrm{~A}-2 \mathrm{H}-4,140-150$ & 9.0 & 2.1 & 10 & 0.20 \\
\hline $135-838 \mathrm{~A}-3 \mathrm{H}-4,140-150$ & 19.0 & 2.1 & 4 & 0.15 \\
\hline $135-838 \mathrm{~A}-4 \mathrm{H}-4,140-150$ & 28.7 & 4.7 & 16 & 0.39 \\
\hline $135-838 \mathrm{~A}-6 \mathrm{H}-4,98-100$ & 47.2 & 4.7 & 3 & 0.39 \\
\hline $135-838 \mathrm{~A}-8 \mathrm{H}-4,140-150$ & 66.7 & 4.7 & 10 & 0.39 \\
\hline $135-838 \mathrm{~A}-10 \mathrm{H}-2,90-92$ & 82.1 & 17.7 & 11 & 1.87 \\
\hline $135-838 \mathrm{~A}-11 \mathrm{H}-5,94-96$ & 96.2 & 17.7 & 13 & 2.10 \\
\hline $135-838 \mathrm{~A}-1 \mathrm{H}-2,140-150$ & 3.0 & 0.9 & 2 & 0.06 \\
\hline $135-838 \mathrm{~A}-2 \mathrm{H}-4,140-150$ & 10.5 & 0.9 & 3 & 0.04 \\
\hline $135-838 \mathrm{~A}-3 \mathrm{H}-4,140-150$ & 20.0 & 5.1 & 2 & 0.24 \\
\hline $135-838 \mathrm{~A}-4 \mathrm{H}-5,140-150$ & 31.0 & 5.1 & 16 & 0.33 \\
\hline $135-838 \mathrm{~A}-5 \mathrm{H} 4,57-59$ & 38.1 & 5.1 & 6 & 0.32 \\
\hline $135-838 \mathrm{~A}-6 \mathrm{H}-4,140-150$ & 48.5 & 5.1 & 4 & 0.36 \\
\hline $135-838 \mathrm{~A}-7 \mathrm{H}-4,108-110$ & 57.6 & 5.1 & 3 & 0.33 \\
\hline $135-838 \mathrm{~A}-8 \mathrm{H}-4,140-150$ & 67.5 & 5.1 & 2 & 0.34 \\
\hline 135-838A-9H-3, 10- 12 & 74.1 & 88.2 & 40 & 5.86 \\
\hline $135-838 \mathrm{~A}-10 \mathrm{H}-5,140-150$ & 88.0 & 88.2 & 74 & 6.51 \\
\hline 135-838A-15X-1, 39-41 & 128.9 & 88.2 & 91 & 5.58 \\
\hline $135-838 A-18 X-c c, 29-31$ & 157.9 & 88.2 & 53 & 6.47 \\
\hline 135-838A-20X-cc, $1-3$ & 176.5 & 88.2 & 198 & 8.68 \\
\hline $135-838 A-21 X-c c, 12-14$ & 186.3 & 88.2 & 155 & 36.6 \\
\hline
\end{tabular}



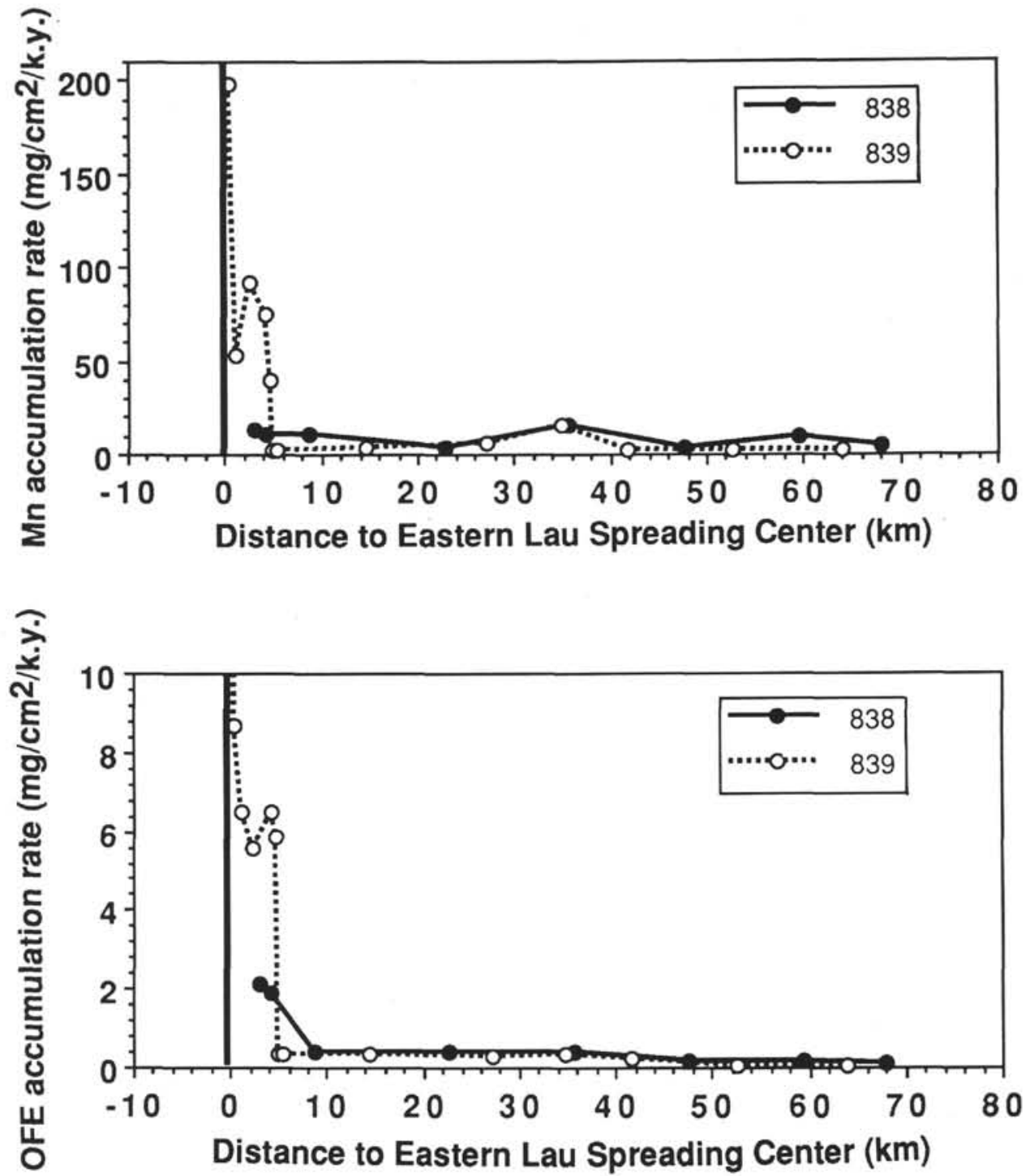

Figure 19. Manganese and ore-forming element (OFE) accumulation rates vs. distance to the Eastern Lau Spreading Center at Sites 838 and 839 (central area of the Lau Basin).

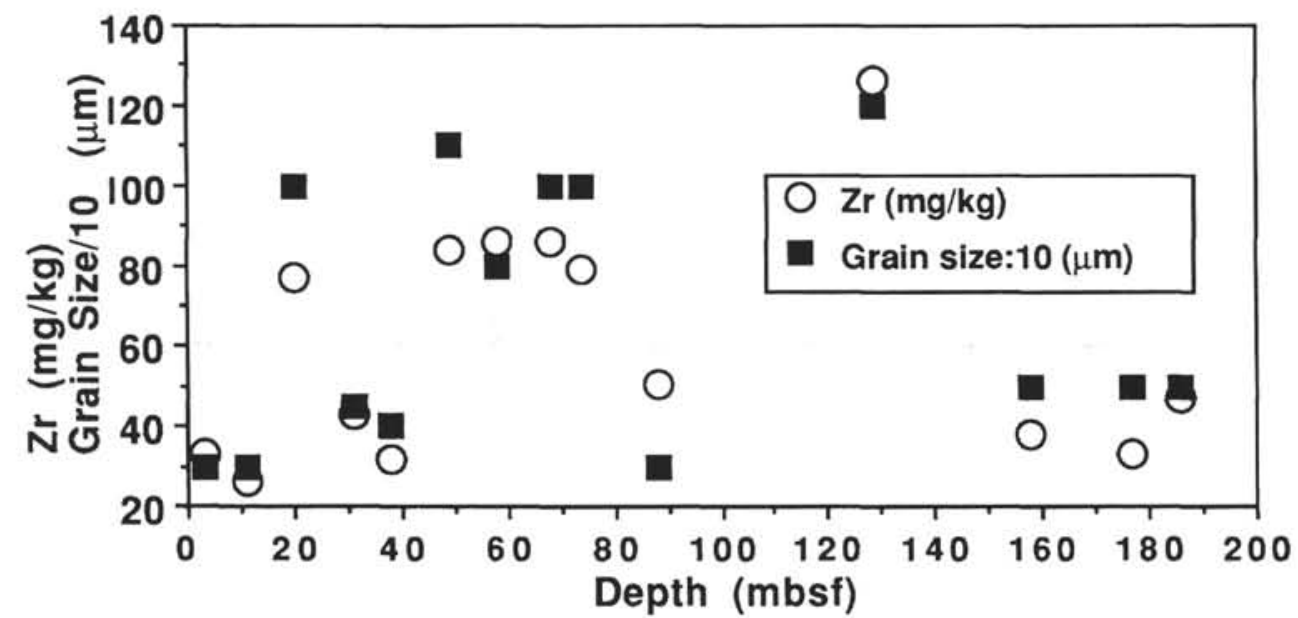

Figure 20. Zr and maximum grain size (divided by 10 ) of volcaniclastic ash layers and epiclastic turbidites vs. depth for all samples from Hole 839A. 
Table 5. Average values of the accumulation rates and sedimentation rates for Holes $834 \mathrm{~A}, 835 \mathrm{~A}, 838 \mathrm{~A}$, and $839 \mathrm{~A}$.

\begin{tabular}{|c|c|c|c|c|c|c|c|c|}
\hline & \multicolumn{7}{|c|}{$\begin{array}{c}\text { Accumulation rates } \\
\text { (mg/cm2/ky) }\end{array}$} & $\begin{array}{c}\text { Sedimentation } \\
\text { rate } \\
(\mathrm{cm} / \mathrm{ky})\end{array}$ \\
\hline HOLES & CF & NCF & MnO & Fe2O3 & HF & VAMF & TF & \\
\hline $135-834 \mathrm{~A}$ & 706 & 528 & 29 & 40 & 70 & 458 & 1234 & 3.5 \\
$135-835 \mathrm{~A}$ & 1290 & 683 & 36 & 51 & 87 & 596 & 1973 & 6.4 \\
$135-838 \mathrm{~A}$ & 342 & 534 & 10 & 14 & 24 & 510 & 876 & 3.4 \\
$135-839 \mathrm{~A}$ & 273 & 1255 & 6 & 9 & 15 & 1240 & 1528 & 4.1 \\
\hline
\end{tabular}

Notes: $\mathrm{CF}=$ carbonate fraction, $\mathrm{NCF}=$ noncarbonate fraction, $\mathrm{HF}=$ hydrothermal fraction, $\mathrm{VAMF}=$ volcanogenic and authigenic material fraction, and $\mathrm{TF}=$ total fraction $($ total sediment). For calculation see text.

in Holes 838A and 839A (central area of the Lau Basin). Diagenetic reactions were favored in a medium characterized by a high accumulation rate of small particles. In such a system, diffusivity is limited and reactive surface is high. On the other hand, diagenetic reactions were prevented in a reworked medium where the specific surface area is low because of coarser grain sizes.

\section{CONCLUSION}

Comparison between the content of the solid phases and the major chemical composition of the studied samples allows three classes of samples to be characterized. They are calcite-rich sediments with $\mathrm{CaO}$ averaging about $42 \mathrm{wt} \%$, calcite-volcanic detritus mixed sediments, and volcanogenic-rich sediments with $\mathrm{SiO}_{2}$ up to $42 \mathrm{wt} \%$.

Higher $\mathrm{Mg} / \mathrm{Ca}, \mathrm{Al} / \mathrm{Si}$, and $\mathrm{Fe} / \mathrm{Mn}$ atomic ratios determined in Class 3 samples strongly suggest an authigenesis of $\mathrm{Fe}-\mathrm{Mg}$ rich clay in older sediments of the central area of the Lau Basin. Phillipsite occurrences in sediments from the northern area (Sites 834-835) probably reflect alteration of volcanogenic material at a grade higher than that in sediments from the central area (Sites 836-839) of the Lau Basin, where $\mathrm{Fe}-\mathrm{Mg}$ rich clay seems to be the main authigenic mineral. High correlation between $\mathrm{Sc}$ and $\mathrm{Al}$ suggests that Sc-bearing authigenic clay occurs in all holes studied. This authigenic clay has probably incorporated $\mathrm{Cr}$ and other transitional elements.

Higher $\mathrm{Sr} / \mathrm{Ca}, \mathrm{Mn} / \mathrm{Ca}, \mathrm{P}_{2} \mathrm{O}_{5} / \mathrm{Y}$, and $\mathrm{Cr} / \mathrm{Ti}$ atomic ratios and enhanced concentrations of OFE and $\mathrm{Mn}$ in samples from Sites 834 and 835 indicate a greater content of the hydrothermal fraction in the northern Lau Basin sediments. Distribution of the accumulation rates of $\mathrm{Mn}$ and $\mathrm{OFE}$ as a function of distance to the ridge shows that hydrothermal precipitates spread over the entire Lau Basin and are also concentrated near the ridge. Therefore, far from the spreading centers, hydrothermal precipitates accumulate predominantly in the northern rather than in the central area of the Lau Basin.

Comparison between the accumulation rates of the hydrothermal fraction and other sedimentary fractions clearly defines the following two sedimentary environments: (1) the northern area, where carbonate and hydrothermal fractions accumulate predominantly; and (2) the central area, characterized by accumulation of a coarser grained volcanogenic fraction. Sediment ponding in the northern area and selective reworking in the central area are possibly responsible for the formation of these two sedimentary domains in the Lau Basin.

\section{ACKNOWLEDGMENTS}

G.B. would like to thank P. Larque (Centre de Géochimie de la Surface) for his careful XRD determinations, and J. Samuel and R. Rouault (Centre de Géochimie de la Surface) for their analytical determinations of major and trace chemical composition. G.B. would like to thank all participants of Leg 135 ODP for a stimulating experience. J. Allan, R.W. Murray, and E.A. Burton are also thanked for reviewing early drafts. This research was supported by INSU Grant GEO 91 3922, "Geosciences marines" (GB).

\section{REFERENCES ${ }^{*}$}

Baker, P.A., Gieskes, J.M., and Elderfield, H., 1982. Diagenesis of carbonates in deep-sea sediments: evidence from $\mathrm{Sr}^{2+} / \mathrm{Ca}^{2+}$ ratios and interstitial dissolved $\mathrm{Sr}^{2+}$ data. J. Sediment. Petrol., 52:71-82.

Besnus, Y., and Rouault, R., 1973. Une méthode d'analyse de roches au spectromètre d'arc à lecture directe par un dispositif d'électrode rotative. Analusis, 2:111-116.

Blanc, G., 1987. Géochimie de la Fosse Atlantis II (Mer Rouge): evolution spatio-temporelle et rôle de l'hydrothermalisme. Publ. Miss. Rech. T.A.A.F., 85-05:212.

Clauer, N., Stille, P., Bonnot-Courtois, C., and Moore, W.S., 1984. Nd-Sr isotopic and REE constraints on the genesis of hydrothermal manganese crusts in the Galapagos. Nature, 311:743-745.

Dymond, J., 1981. The geochemistry of Nazca Plate sediments: an evaluation of hydrothermal, biogenic, detrital, and hydrogenous sources. In Kulm, L.D., Dymond, J., Dasch, D.M., and Hussong, D.M. (Eds.), Nazca Plate: Crustal Formation and Andean Convergence. Mem.-Geol. Soc. Am., 154:133-174.

Edmond, J.M., von Damm, K.L., McDuff, R.E., and Measures, C.I., 1982. Chemistry of hot springs on the East Pacific Rise and their effluent dispersal. Nature, 297:187-191.

Elderfield, H., 1977. The form of manganese and iron in marine sediments. In Glasby, G.P. (Ed.), Marine Manganese Deposits: Amsterdam (Elsevier), Elsevier Oceanogr. Ser., 15:270-289.

Fouquet, J.P., von Stackelberg, U., and Shipboard Scientific Party, 1990. Hydrothermal activity in the Lau Basin. Eos, 71:678-679.

Fouquet, Y., von Stackelberg, U., and Charlou, J.L., Donval, J.P., Foucher, J.P., Erzinger, J., Herzig, P., Mühe, R., Wiedicke, M., Soakai, S., and Whitechurch, H., 1991. Hydrothermal activity in the Lau backarc basin: sulfides and water chemistry. Geology, 19:303-306.

Graham, D.W., Bender, M.L., Williams, D.F., and Keigwin, L.D., Jr., 1982. Strontium-calcium ratios in Cenozoic planktonic foraminifera. Geochim. Cosmochim. Acta, 46:1281-1292.

Heath, G.R., 1981. Metalliferous-sediment deposition in time and space: east Pacific Rise and Bauer Basin, northern Nazca Plate. In Kulm, L.D., Dymond, J., Dasch, E.J., and Hussong, D. (Eds.), Nazca Plate: Crustal Formation and Andean Convergence. Mem.-Geol. Soc. Am., 154:175-197.

Heath, G.R., and Dymond, J., 1977. Genesis and transformation of metalliferous sediments from the East Pacific Rise, Bauer Deep, and Central Basin, Northwest Nazca Plate. Geol. Soc. Am. Bull., 88:723-733.

Hooton, D.H., and Giorgetta, N.E., 1977. Quantitative X-ray diffraction analysis by a direct calculation method. X-Ray Spectrom., 6:2-5.

Kastner, M., and Stonecipher, S.A., 1978. Zeolites in pelagic sediments of the Atlantic, Pacific, and Indian oceans. In Sand, L.B., and Mumpton, F.A. (Eds.), Natural Zeolites: Occurrence, Properties, Use: New York (Pergamon Press), 199-218.

Kunzendorf, H., Walter, P., Stoffers, P., and Gwozdz, R., 1990. Rare and precious element geochemistry of sediments from the Lau Basin. Geol. Jahrb., Reihe D, 92:263-278.

Lyle, M., 1981. Formation and growth of ferromanganese oxides on the Nazca Plate. In Kulm, L.D., Dymond, J., Dasch, E.J., and Hussong, D. (Eds.),

\footnotetext{
Abbreviations for names of organizations and publication titles in ODP reference lists follow the style given in Chemical Abstracts Service Source Index (published by American Chemical Society).
} 
Nazca Plate: Crustal Formation and Andean Convergence. Mem.-Geol. Soc. Am., 154:269-293.

Lyle, M., Owen, R.M., and Leinen, M., 1986. History of hydrothermal sedimentation at the East Pacific Rise, $19^{\circ}$ S. In Leinen, M., Rea, D.K., et al., Init. Repts. DSDP, 92: Washington (U.S. Govt. Printing Office), 585-596.

Mangini, A., and Stoffers, P., 1990. A high resolution ${ }^{230}$ Th depth profile in a piston core from the Southern lau Basin. Geol. Jahrb., Reihe D, 92:255-261.

Marchig, V., and Erzinger, J., 1986. Chemical composition of Pacific sediments near $20^{\circ} \mathrm{S}$ : changes with increasing distance from the East Pacific Rise. In Leinen, M., Rea, D.K., et al., Init. Repts. DSDP, 92: Washington (U.S. Govt. Printing Office), 371-381.

Marchig, V., Gundlach, H., Möller, P., and Schlei, F., 1982. Some geochemical indicators for discrimination between diagenetic and hydrothermal metalliferous sediments. Mar. Geol., 50:241-256.

Marchig, V., Gundlach, H., and Schmitz, W., 1990. Geochemical studies of sediment cores from the North Fiji Basin. Geol. Jahrb., Reihe D, 92:189-208.

McMurtry, G.M., Veeh, H.H., and Moser, C., 1981. Sediment accumulation rate patterns on the northwest Nazca Plate. In Kulm, L.D., Dymond, J., Dasch, E.J., and Hussong, D. (Eds.), Nazca Plate: Crustal Formation and Andean Convergence. Mem.-Geol. Soc. Am., 154:211-249.

Parson, L., Hawkins, J., Allan, J., et al., 1992. Proc. ODP, Init. Repts., 135: College Station, TX (Ocean Drilling Program).

Riech, V., 1990. Calcareous oozes, volcanic ashes and metalliferous sediments in the Quaternary of the Lau and North Fiji Basins. Geol. Jahrb., Reihe D, 92:109-162.

Ruhlin, D.E., and Owen, R.M., 1986. Factors influencing the rare earth element composition of hydrothermal precipitates, East Pacific Rise. In Leinen, M., Rea, D.K., et al., Init. Repts. DSDP, 92: Washington (U.S. Govt. Printing Office), 383-389.
Samuel, J., Rouault, R., and Besnus, Y., 1985. Analyse multiélémentaire standardisée des matériaux géologiques en spectrométrie d'emission par plasma à couplage inductif. Analusis, 13:312-317.

Schrader, E.L., Furbish, W.J., Mattey, D., and May, J.A., 1980. Geochemistry and carbonate petrology of selected sediment samples from Deep Sea Drilling Project Leg 54, Eastern Pacific. In Rosendahl, B.R., Hekinian, R., et al., Init. Repts. DSDP, 54: Washington (U.S. Govt. Printing Office), 319-328.

Von Damm, K.L., Edmond, J.M., Grant, B., Measures, C.I., Walden, B., and Weiss, R.F., 1985. Chemistry of submarine hydrothermal solution at $21^{\circ} \mathrm{N}$. East Pacific Rise. Geochim. Cosmochim. Acta, 49:2197-2220.

von Rad, U., Frenzel, G., and Mühe, R., 1990. Origin and alteration of submarine volcaniclastic rocks from the Lau and North Fiji Basins (SW Pacific, SO-35 Cruise). Geol. Jahrb., Reihe D, 92:341-393.

von Stackelberg, U., von Rad, U., Marchig, V., Muller, P., and Weiser, T., 1990. Hydrothermal mineralization in the Lau and North Fiji Basins. Geol. Jahrb., Reihe D, 92:547-613.

von Stackelberg, U., von Rad, U., and Shipboard Scientific Party, 1988. Active hydrothermalism in the Lau backarc basin (SW-Pacific): first results from the SONNE 48 cruise (1987). Mar. Min., 7:431-442.

Walter, P., Stoffers, P., Glasby, G.P., and Marchig, V., 1990. Major and trace element geochemistry of Lau Basin sediments. Geol. Jahrb., Reihe D, $92: 163-188$.

Date of initial receipt: 31 August 1992

Date of acceptance: 20 May 1993

Ms 135SR-124 